Vol. 11, n² | 2007

Varia

\title{
Enfants et adolescents en prison " ordinaire » : L'incarcération des mineurs en France selon les statistiques pénitentiaires (1880-1945)
}

Élise Yvorel

\author{
(2) OpenEdition \\ Journals \\ Édition électronique \\ URL : https://journals.openedition.org/chs/116 \\ DOI : $10.4000 /$ chs. 116 \\ ISSN : 1663-4837 \\ Éditeur \\ Librairie Droz \\ Édition imprimée \\ Date de publication : 1 décembre 2007 \\ Pagination : 107-138 \\ ISBN : 978-2-600-01224-9 \\ ISSN : 1422-0857
}

Référence électronique

Élise Yvorel, «Enfants et adolescents en prison « ordinaire » : L'incarcération des mineurs en France selon les statistiques pénitentiaires (1880-1945) », Crime, Histoire \& Sociétés / Crime, History \& Societies [En ligne], Vol. 11, n² | 2007, mis en ligne le 01 mars 2011, consulté le 22 mars 2022. URL : http:// journals.openedition.org/chs/116; DOI : https://doi.org/10.4000/chs.116

Ce document a été généré automatiquement le 22 mars 2022

(C) Droz 


\title{
Enfants et adolescents en prison " ordinaire »: L'incarcération des mineurs en France selon les statistiques pénitentiaires (1880-1945)
}

\author{
Élise Yvorel
}

\section{Introduction et présentation de la source}

$1 \quad$ L'historiographie de la répression de la délinquance juvénile ${ }^{2}$ laisse penser que seules les «maisons de correction » ont accueilli les mineurs de justice. L'« effet Mettray » ${ }^{3}$ a été puissant. Les colonies pénitentiaires, institutions originales conçues exclusivement pour les mineurs, ces «bagnes d'enfants", ont accaparé autant l'imaginaire collectif ${ }^{4}$ que les curiosités des chercheurs. Pourtant, en vertu de dispositions législatives complexes, les mineurs pénaux sont nombreux à être incarcérés dans les prisons " ordinaires ", celles aux hauts murs et aux fenêtres à barreaux dans lesquelles il est plus courant d'imaginer un contingent de brigands et de criminels adultes. Jeunes prévenus et jeunes condamnés, garçons et filles de la correction paternelle mais aussi jeunes acquittés pour avoir agi sans discernement et en attente de transfert en colonies agricole, industrielle ou maritime ${ }^{5}$, se sont côtoyés dans la prison parisienne pour enfant de la Petite-Roquette ${ }^{6}$ et dans les maisons d'arrêt de justice et de correction, prisons départementales nombreuses et souvent surpeuplées 7 . C'est le nombre de ces mineurs pénaux détenus effectivement dans ces établissements pénitentiaires, dans ces prisons « ordinaires ", qu'il s'agit de déterminer ici.

2 Au delà des chiffres bruts, du nombre exact de jeunes détenus, il importe également, dans la mesure du possible, de déterminer la répartition des mineurs selon leur catégorie légale (prévenus, condamnés, incarcérés civils), leur âge et leur sexe. Les 
statistiques renseignent en effet sur une certaine vision sociale de la jeunesse, sur l'application de la loi, sur les tendances que traverse la répression pénale de la délinquance juvénile et sur les conditions de détention des jeunes prisonniers. Cependant, les obstacles pour arriver à un degré satisfaisant de précision dans des statistiques pénitentiaires sont nombreux et pas toujours franchissables. Les sources officielles disponibles sont incomplètes et peu linéaires.

3 Les rapports annuels officiels apportent l'illusion de pouvoir obtenir un aperçu général sur une longue période et donnent la garantie d'être régulièrement édités et relativement aisés d'accès. Comme il n'est guère question d'analyser ni l'évolution de la délinquance juvénile, ni la politique pénale, Le Compte général de l'administration de la justice criminelle n'a pas été retenu pour ce travail. En effet, cette source essentielle pour qui étudie la délinquance juvénile ou plutôt, le fonctionnement de la justice pénale des mineurs et le recours par les magistrats à l'enfermement, est inutilisable pour comptabiliser le nombre d'enfants qui passent en maison d'arrêt. Sur la question de l'incarcération des mineurs au XXe siècle, seuls sont exploitables les différents volumes de Statistiques pénitentiaires proposés annuellement au garde des Sceaux de 1855 à 1945, période à laquelle ils prennent le nom de Rapport annuel de fonctionnementde l'Administration pénitentiaire et délaissent, à cette occasion, quelque peu les données chiffrées. Cette série, comme toutes autres, possède ses avantages, ses inconvénients et surtout ses pièges.

4 Précisons qu'il a été impossible de rassembler toute la série. Le tome consacré à l'année 1936 s'est avéré soit manquant soit incommunicable. Tous les autres volumes, de 1880 à 1945, sont accessibles avec, toutefois, une interruption éditoriale de 1940 à 1944, conséquence d'une période de confusion dans les établissements pénitentiaires, souvent réquisitionnés par les Allemands. Excepté ces lacunes et une rupture considérable en 1910, sur laquelle nous reviendrons, l'organisation intérieure des volumes subit peu de modifications sur les soixante-six années envisagées. Ces ouvrages traitent principalement des situations matérielles et humaines de trois catégories de détenus en fonction de leurs situations légales pénales, chacune liée à un type d'établissement: l'exécution des longues peines en maison centrale, celle des courtes peines en maison d'arrêt et l'éducation correctionnelle en colonies pénitentiaires. Ces statistiques pénitentiaires sont formées d'une grande quantité de tableaux à multiples entrées qui traitent aussi bien de l'état général de la population détenue que des détails des conditions de détention.

5 C'est dans la partie consacrée aux maisons d'arrêt, de justice et de correction que se trouvent les informations les plus éclairantes, mais néanmoins assez restreintes, sur la situation des mineurs incarcérés. En effet, l'âge des détenus est rarement une entrée choisie et enfants et adultes sont confondus sous la dénomination générale d'« Hommes et jeunes garçons" et de "Femmes et jeunes filles». Cela empêche naturellement toute possibilité de distinguer les mineurs des majeurs. Toutefois, les tableaux $n^{\circ} I I$ de cette partie dévolue aux maisons d'arrêt, font exception puisqu'ils traitent de la «répartition de la population incarcérée pendant l'année d'après la situation légale » et isolent les mineurs. Il est donc possible de connaître l'évolution quantitative de l'ensemble des jeunes détenus incarcérés en maisons d'arrêt mais également de déterminer leur sexe et leur condition de prévenu, de condamné à une courte peine privative de liberté ou d'incarcérés civils. Dans ces tableaux, un bouleversement dans la méthode de comptabilisation de la population pénale survient 
en 1909-1910. Avant cette date charnière, l'Administration pénitentiaire compte le nombre de détenus présents dans les maisons d'arrêt au 31 décembre de l'année concernée. Elle distingue les majeurs des mineurs et, parmi ces derniers, elle différencie les « garçons » et les « filles », les jeunes incarcérés « par voie de correction paternelle », les " prévenus, accusés, jugés en appel ou en pourvoi », les « condamnés à un emprisonnement de six mois et au-dessous» et les «jugés attendant leur transfèrement». À partir de 1910, les mêmes tableaux $n^{\circ}$ II comptent non plus le «stock » mais le «flux » des détenus, c'est-à-dire le nombre de détenus qui sont passés en maison d'arrêt au cours de l'année ${ }^{8}$. Il est donc impossible de comparer les tableaux nII établis de 1880 à 1909 avec ceux de 1910 à 1945. Les courbes effectuées à partir de ces données chiffrées et les analyses statistiques qui suivent sont, par conséquent, divisées en fonction de cette donne imposée par la source officielle. De plus, les informations sur la situation pénale des jeunes se précisent. Outre la différenciation des filles et des garçons, la catégorie "mineurs prévenus » est divisée en "prévenus et accusés » et « condamnés qui ont fait appel ou sont en pourvoi »; celle des « condamnés définitivement » distingue les " pupilles condamnés à un emprisonnement de six mois et au-dessous » et les "pupilles jugés attendant leur transfèrement »; enfin, celle des « internés » sépare les «pupilles venant de l'Assistance publique ou pour correction paternelle $»^{9}$.

6 Compte tenu de ces contraintes, il est possible, dans un premier temps, d'établir des courbes de mouvement général de l'ensemble de la population des mineurs incarcérés en maison d'arrêt, de justice et de correction au 31 décembre jusqu'en 1909, puis écroués pendant l'année jusqu'en 1945, avec une distinction entre les garçons et les filles. Dans un second temps, c'est la situation légale de ces jeunes détenus et la répartition sexuée des prisonniers qui est examinée, toujours en considérant la scission chronologique induite par la source. Ce travail permet de mesurer la part des prévenus et des condamnés gardés dans les prisons départementales mais également celle prise par les jeunes écroués par voie de correction paternelle ou en vertu de la loi de 1904 sur les pupilles vicieux de l'Assistance publique. Sporadiquement, quelques données chiffrées sur l'enfermement en «maisons de correction» publiques et privées, étayeront la démonstration à titre de comparaison. Toutefois, comme chaque fois qu'il est fait appel aux statistiques, il faut manier les chiffres avec précaution : l'étude des statistiques pénitentiaires ne donne pas d'indications sur le nombre de mineurs délinquants ni sur le nombre de jugements, mais uniquement sur le nombre des mineurs effectivement en détention qu'ils soient, ou non, jugés et condamnés. Ces données permettent simplement de proposer un ordre de grandeur à l'incarcération des mineurs et autorisent à soulever quelques interrogations ou hypothèses. 
Document 1 : Reproduction d'extraits des tableaux $n^{\circ}$ II des rapports de Statistiques pénitentiaires des années 1895 et 1911

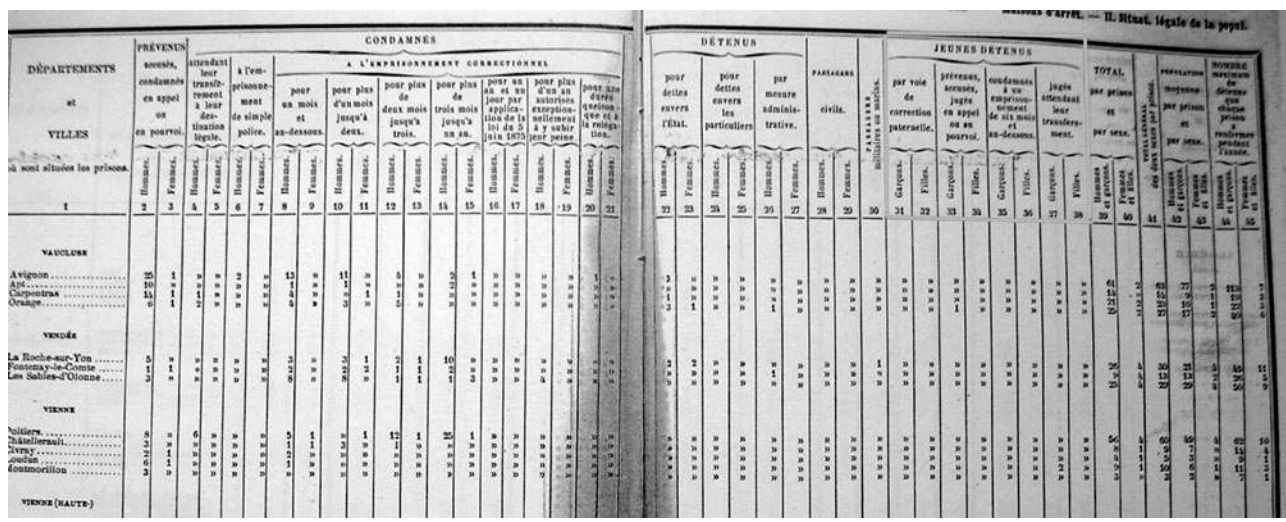

Tableau n II en 1895 : division en terme de situation légale de la catégorie des « jeunes détenus »

\section{I - Les mouvements généraux avant 1945}

\section{Les mineurs détenus au 31 décembre de 1880 à 1909 : une nouvelle conception de l'enfance}

7 De 1880 à 1909, les statistiques annuelles de l'Administration pénitentiaire indiquent le nombre de mineurs détenus en maison d'arrêt, de justice et de correction au 31 décembre de l'année. Cela ne permet guère d'examiner directement le recours à l'incarcération mais d'établir les évolutions et les taux de détention. Seules les données en flux, où sont dénombrées les entrées au cours de l'année, permettent une évaluation de la proportion de mineurs ayant fait un séjour carcéral. L'écart entre les données en flux et les données en stock dépend du temps de séjour moyen : plus les séjours sont courts, plus le taux de rotation est important et plus le nombre de jeunes détenus au 31 décembre est éloigné du nombre de jeunes détenus passés par la prison pendant l'année de référence. La courbe infra représente donc l'ensemble des mineurs pénaux, garçons et filles, détenus dans les maisons d'arrêt, de justice et de correction au 31 décembre de chaque année, de 1880 à 1909. 
Graphique 1 : Ensemble des mineurs détenus en maison d'arrêt, de justice et de correction au 31 décembre de l'année (1880-1909)

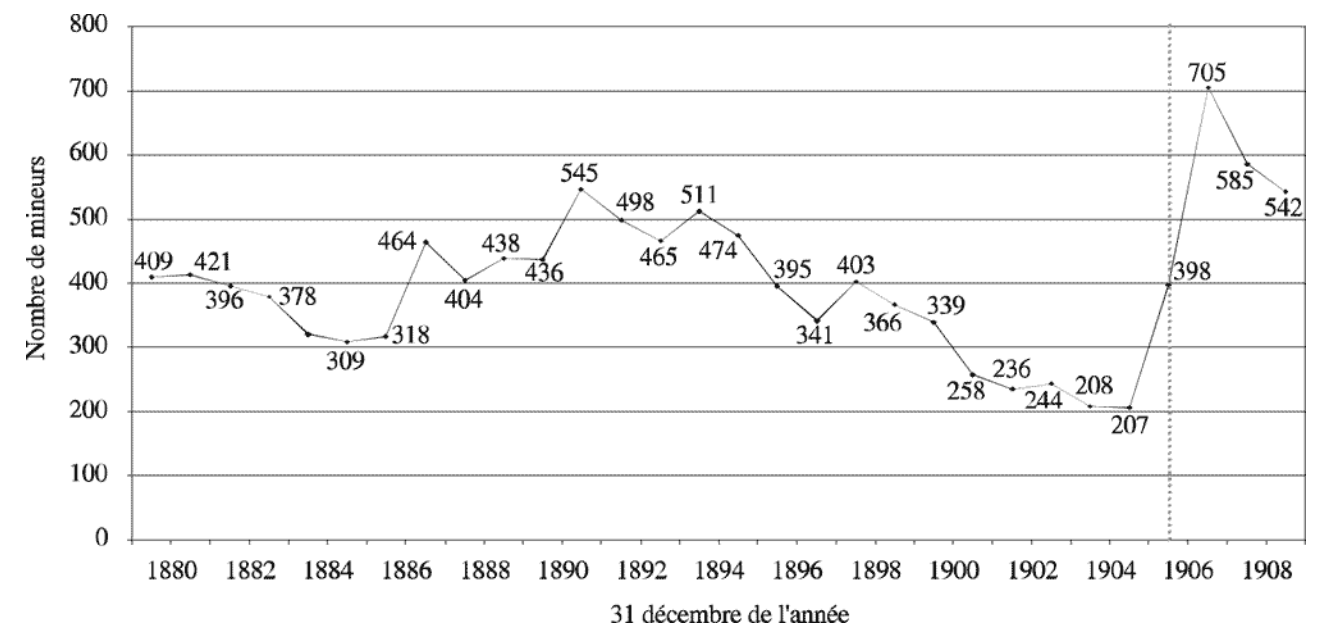

8 Sur l'ensemble de la période, jusqu'en 1905, la tendance de la courbe est globalement à la baisse malgré des fluctuations relativement importantes. Le pic de 1887 rompt avec une tendance descendante depuis 1880 . Le nombre de mineurs détenus au 31 décembre passe en effet de 318 à 464 et dépasse ainsi le chiffre le plus important des sept années précédentes, à savoir, 412 en 1881. En 1888, le «stock » des jeunes accuse une baisse de 60 unités, puis la courbe prend une tendance ascendante jusqu'en 1891, année où le nombre d'adolescents détenus est le plus élevé de la période 1880-1906 avec 545 enfants. Par la suite, la tendance de la courbe est constamment à la décrue jusqu'en 1906 malgré les deux soubresauts de 1894 et de 1898. À partir de 1907, le nombre de jeunes incarcérés subit une croissance particulièrement importante. De 318 en 1906, et c'est déjà une augmentation sensible par rapport à 1905 où 207 mineurs étaient incarcérés, il double pratiquement et passe à 705 en 1907. Cette brusque augmentation s'explique simplement par la prise en compte d'une nouvelle catégorie de jeunes, ceux de 16-18 ans, induite par la loi du 12 avril 1906 « modifiant les articles 66 et 67 du code pénal ${ }^{10}, 340 \mathrm{du}$ Code d'instruction criminelle, et fixant la majorité pénale à l'âge de 18 ans ». Les mineurs pénaux regroupent désormais tous les enfants de moins de 18 ans et non plus seulement ceux de moins de 16 ans $^{11}$. Les statistiques prennent évidemment ce changement en considération et il est donc impossible ici de comparer le nombre de jeunes détenus incarcérés de 1880 à 1906 avec ceux de 1907 à $1909^{12}$. De plus, il n'est pas précisé dans le rapport quels sont les mineurs comptés en 1906 (il en est d'ailleurs de même pour les effectifs des colonies pénitentiaires). Les 398 individus comptabilisés cette année-là comprennent-ils l'ensemble des adolescents de moins de 16 ans ou l'ensemble de ceux de moins de 18 ans ? Ou encore les adolescents de moins de 16 ans jusqu'en avril 1906 puis tous ceux de moins de 18 ans après l'adoption de la loi ? Cette dernière interprétation pourrait expliquer la brusque remontée du nombre de mineurs incarcérés au 31 décembre 1906 (398) par rapport à 1905 (207), et qui n'est cependant pas aussi conséquente que celle indiquée pour 1907 (705). Cette année ambiguë écartée, un constat demeure : les adolescents de 16-18 ans semblent au moins aussi nombreux à être incarcérés que leurs benjamins. En effet, la moyenne des mineurs des moins de 16 ans détenus à la fin des années de 1880 à 1905 est de 377 alors que 705 mineurs de moins de 18 ans sont incarcérés le 31 décembre 1907, soit 328 personnes supplémentaires. Il est probable que ces jeunes gens soient majoritairement âgés de 16 à 18 ans. Par la suite cependant, la courbe semble de nouveau s'orienter à la baisse. 
9 La tendance descendante au début de la période d'étude met en évidence une diminution du recours à l'incarcération par les tribunaux. Ce phénomène peut s'expliquer, en grande partie, par un changement des conceptions de la jeunesse dans la société civile. L'émergence du courant criminologique français qui se penche alors sur les questions de l'enfance à problème met en avant les causes sociales de la délinquance telles l'alcoolisme et le manque d'éducation ${ }^{13}$. Le Vicomte d'Haussonville, note déjà en 1875 que « la misère et la mauvaise éducation, sont les causes principales de la dépravation chez l'enfant $»^{14}$. Les lois sur la scolarité de Jules Ferry en 1882 sont d'ailleurs assez emblématiques d'une évolution des mentalités ${ }^{15}$. Ce nouveau rapport aux jeunes semble bien toucher les juges qui évitent davantage de faire appel à l'incarcération pour cette catégorie judiciaire, au profit d'autres structures moins répressives ${ }^{16}$. C'est l'analyse de plusieurs historiens dont Jean-Claude Vimont :

Une notable partie des enfants étant victime de leur environnement familial, on

décide qu'il convient de les protéger et de leur éviter la prison en les confiant à des

sociétés charitables de patronage $\mathrm{e}^{17}$.

En revanche, aucune explication dans le contexte politique et juridique ne semble pouvoir justifier le pic de 1887 (plus $45,9 \%$ par rapport à 1886). Toutefois, la tendance à la progression qui s'ensuit, jusqu'en 1894, trouve peut-être une explication dans la politique de laïcisation républicaine. Cette dernière provoque en effet la fermeture de la majorité des établissements privés, souvent religieux ${ }^{18}$, alors que les colonies pénitentiaires d'État ne sont guère nombreuses. Les magistrats qui ne veulent pas remettre les enfants à leur famille, ont à disposition un nombre très limité d'institutions d'éducation correctionnelle ${ }^{19}$. Il est donc possible qu'un certain nombre d'entre eux ait, à défaut de mieux, recours à de courtes peines privatives de liberté en prison « ordinaire». Cependant, les colonies publiques se spécialisent, durcissent leur régime de discipline, et les enfants y sont finalement largement envoyés ${ }^{20}$. La tendance à la baisse de 1894 à 1905 peut, à nouveau, être imputée à l'évolution des mentalités face à la protection de l'enfance ${ }^{21}$. En effet, une politique de prévention et d'intervention auprès des familles se développe ${ }^{22}$ et aboutit finalement aux lois de $1889^{23}$ sur la protection des enfants maltraités ou moralement abandonnés et de $1898^{24}$ sur la répression des violences, voies de fait, actes de cruauté et attentats commis envers les enfants. L'instauration de la loi sur le sursis simple du 26 mars 1891 contribue, elle aussi, à un allègement certain de la population carcérale ${ }^{25}$. Elle permet en effet aux juges d'accorder un sursis aux délinquants primaires qui encombrent les prisons départementales, catégorie à laquelle appartiennent de nombreux mineurs. Quant aux soubresauts de 1894 (plus 9,9\% par rapport à 1893) et de 1898 (plus 18,2\% par rapport à 1897), ils ne trouvent guère d'explication dans les politiques d'ensemble de l'État. La loi de 1904 sur les pupilles vicieux de l'Assistance publique quant à elle, ne semble pas avoir de conséquence immédiate sur le nombre de mineurs incarcérés en fin d'année ${ }^{26}$. Les années 1904 et 1905 représentent en effet le chiffre le plus bas de jeunes détenus (respectivement 206 et 207). Peut-être que des justifications à ces variations résident dans le traitement judiciaire différencié des garçons et des filles. La courbe infra distingue la tendance à l'incarcération pour les jeunes hommes et pour les jeunes femmes au 31 décembre de chaque année. 
Graphique 2 : Garçons et filles incarcérés en maison d'arrêt, de justice et de correction au 31 décembre de l'année (1880-1909)

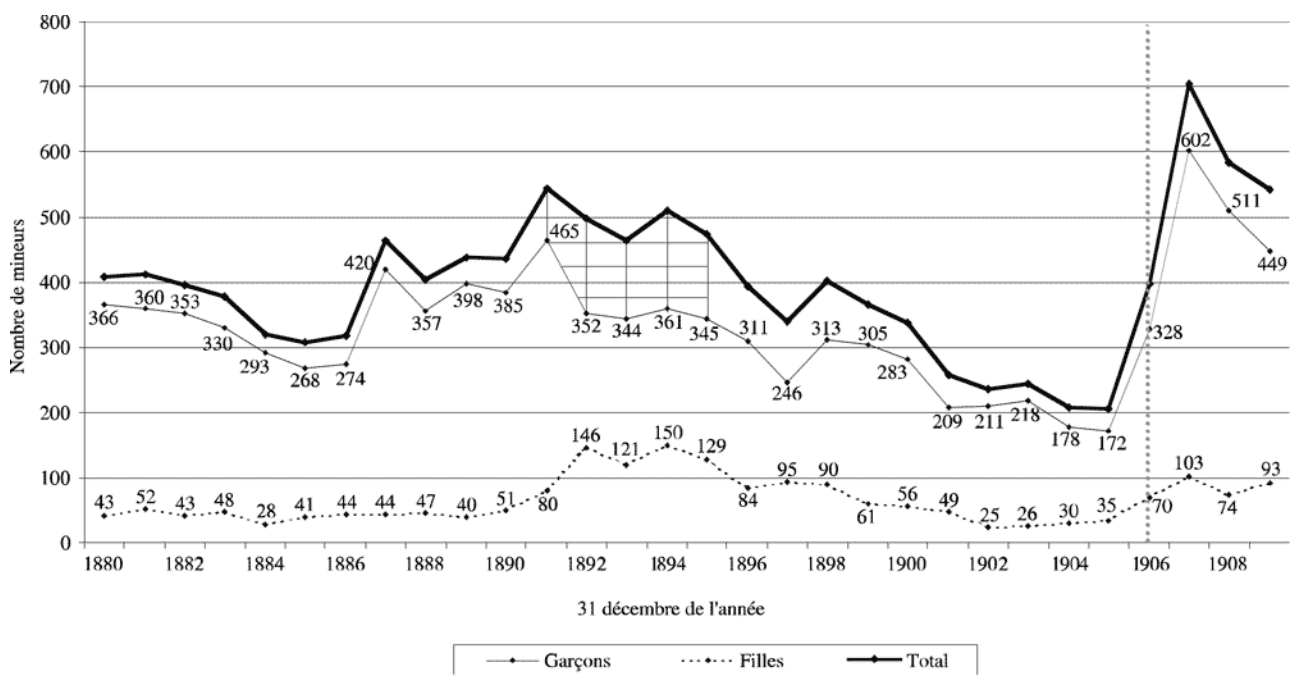

11 Après mise en perspective, le premier constat de rigueur, est la similitude entre la courbe qui représente l'ensemble des mineurs incarcérés au 31 décembre de 1880 à 1909 et celle de l'incarcération des garçons. Le mouvement général est à peu près constamment le même pour les deux courbes. En revanche, la courbe qui représente les filles semble avoir une évolution plus indépendante. De fait, les explications fournies pour la tendance générale concernent essentiellement les garçons. Il serait, en outre, certainement utile de mentionner d'éventuels événements conjoncturels pour justifier tel pic ou telle tendance. Ainsi, l'ouverture de la maison d'éducation agricole de Montesson dans la région parisienne en 1896 contribue-t-elle, peut-être, à vider la Petite-Roquette d'une partie de ses effectifs et à accentuer la tendance à la décrue de la courbe à cette période. De même, des difficultés momentanées dans le système de transfert des jeunes des maisons d'arrêt jusqu'aux colonies où ils doivent être retenus et éduqués ${ }^{27}$, peuvent jouer sur le «stock » au 31 décembre. Cette théorie est d'autant plus plausible que de nombreux mineurs de justice passent par la prison avant que leur non-discernement soit établi et qu'ils soient éventuellement, de la sorte, destinés à rejoindre une "maison de correction $»^{28}$. Cependant, ces phénomènes, nombreux et mouvants, sont difficiles à repérer. De plus, leur influence réelle sur la détention dans les prisons départementales n'est pas mesurable. Il est seulement possible ici d'émettre des hypothèses.

La courbe de la détention des filles, quant à elle, révèle plusieurs pratiques des tribunaux face à la délinquance féminine. La plus évidente est que les magistrats ordonnent moins de mise en détention pour les filles que pour les garçons. Au delà de cet état de fait, des différences dans les tendances des courbes masculine et féminine méritent quelques explications. De 1880 à 1890, le nombre de jeunes filles incarcérées au 31 décembre est à peu près constant et ne dépasse pas les 52 unités, alors que celui des jeunes hommes est en baisse jusqu'en 1886 avant d'amorcer une hausse significative qui prend fin en 1891. En revanche, la quantité de demoiselles détenues augmente brusquement en l'espace de trois ans (multipliée par plus de trois et demi de 1889 à 1892) puis elle reste élevée jusqu'en 1895 avant l'amorce d'une diminution continue qui prend fin en 1905. Certes, les chiffres considérés sont faibles mais, même proportionnellement aux garçons, la progression reste conséquente. C'est uniquement 
sur cette courte période d'évolution contraire chez les filles et chez les garçons, de 1891 à 1895 , que le nombre de filles détenues influe significativement sur la tendance générale ${ }^{29}$. Cette augmentation de filles détenues a deux explications plausibles. La première est une peur croissante des maladies vénériennes ${ }^{30}$ qui conduit la police des mœurs à arrêter fréquemment les femmes et jeunes filles qui se prostituent. Or, les mineures péripatéticiennes sont conduites à Saint-Lazare ${ }^{31}$, maison d'arrêt pour femmes qui possède sa propre infirmerie. Les filles y subissent un dépistage de la syphilis et y reçoivent éventuellement des soins, avant d'être libérées ${ }^{32}$. Ces gamines de la rue apparaissent alors dans les registres d'écrou. La seconde explication rejoint la première. Les demoiselles interpellées pour "vagabondage " sont remises, jusqu'en 1886, au couvent de la Madeleine qui prend en charge tout l'effectif en bonne santé de Saint-Lazare ${ }^{33}$. Cependant, en 1887, dans le cadre de sa politique de laïcisation, l'État rompt son contrat avec cet établissement. Les jeunes prostituées comme les filles de la correction paternelle retournent dans les maisons d'arrêt, auxquelles appartient entre autres le quartier spécial pour mineures de Nanterre. L'ouverture, en 1895, de l'École de préservation de Doullens explique la décroissance de la courbe des filles détenues dans les prisons départementales puisque cette institution permet de désengorger en partie Saint-Lazare et Nanterre $^{34}$. La croissance qui s'amorce de nouveau en 1906 peut dépendre, comme pour les garçons, de la loi d'avril 1906 sur la majorité pénale. Néanmoins, le directeur de l'Administration pénitentiaire propose, dans son rapport de fonctionnement de l'année, une autre interprétation :

Le total des femmes [détenues dans des prisons de courtes peines au 31 décembre 1906] paraît élevé, en apparence seulement, si on le compare à celui des hommes. La différence provient du nombre considérable de femmes et de jeunes filles publiques arrêtées à Paris par ordre administratif et par mesure sanitaire, et qui sont remises en liberté après quelques jours de détention à Saint-Lazare ${ }^{35}$.

Il est probable que ces deux facteurs influent sur la hausse de l'effectif des filles mineures incarcérées en 1906 (hausse de 133,3 \%) par rapport à 1904 mais aucun moyen ne permet de mesurer la part de chacun d'entre eux. La tendance d'ensemble de l'incarcération des jeunes est donc peu affectée par la fluctuation du nombre de filles détenues. Comme le montre l'histogramme ci-dessous, celles-ci sont en effet peu nombreuses, par rapport aux garçons, à être retenues en maison d'arrêt : 
Histogramme 1 : Pourcentage des filles détenues en maison d'arrêt, de justice et de correction au 31 décembre, par rapport à l'ensemble des jeunes détenus (1880-1909)

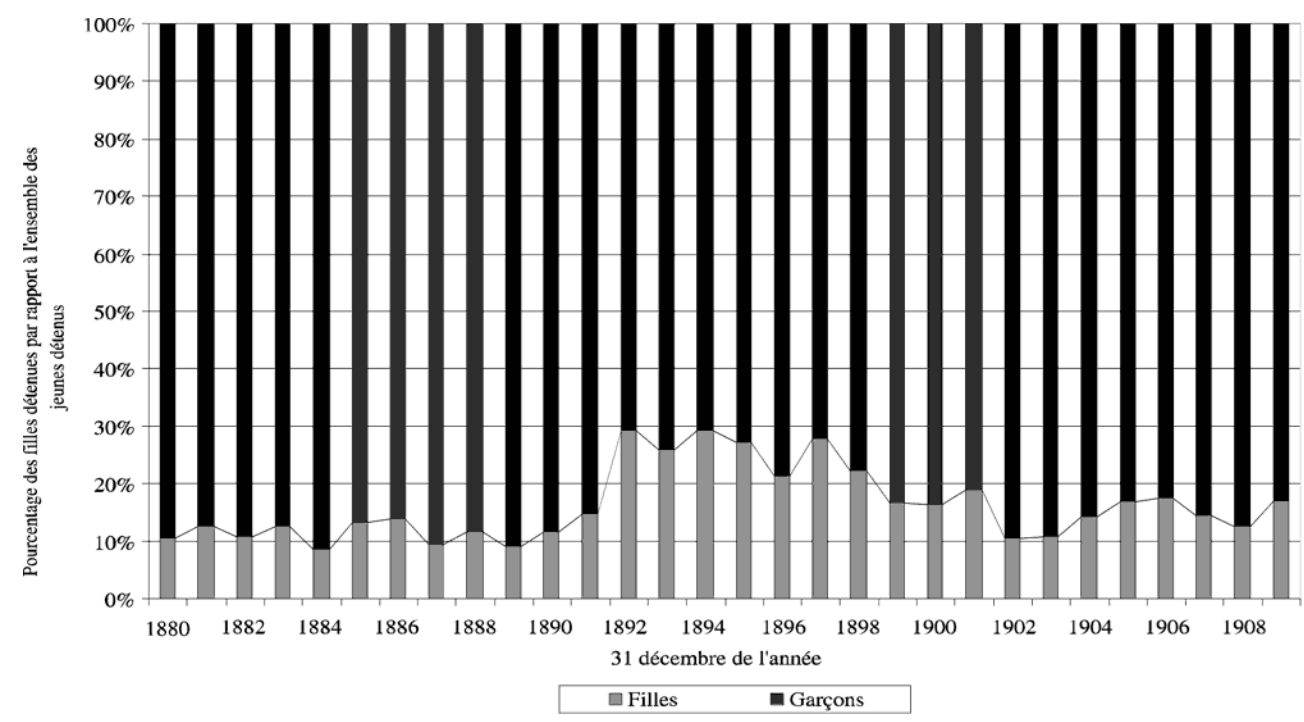

Les filles représentent jusqu'à $29,3 \%$ de l'ensemble des mineurs détenus en 1892 et en 1894 mais cela est exceptionnel sur la période. La part féminine des jeunes détenus est supérieure à $20 \%$ sur sept années, de 1892 à 1898 . De 1880 à 1891 puis de 1899 à la fin de la période, la proportion des filles par rapport aux garçons ne franchit pas cette limite, oscillant entre 8,7 \% en 1884 et $19 \%$ en 1901.

\section{Les entrées en maison d'arrêt des mineurs de 1910 à 1945 : la décrue}

15 À partir de 1910, les volumes des Statistiques pénitentiaires ne décomptent plus les mineurs détenus au 31 décembre de l'année dans les maisons d'arrêt, de justice et de correction mais le nombre d'entrées de mineurs dans les prisons départementales tout au long de l'année. Le flux, par rapport au stock, a un avantage : il est beaucoup plus représentatif du recours à l'incarcération puisqu'il compte les entrées en prisons. Cependant, comme le calcul se fait par catégories pénales, il en résulte un phénomène de double comptage : un jeune incarcéré au mois de janvier est compté comme prévenu ou accusé puis, s'il est jugé et qu'il fait appel, il est comptabilisé une seconde fois en tant que prévenu en appel ou en pourvoi. Il peut être compté une troisième fois s'il est jugé définitivement et condamné, soit comme mineur qui subit une courte peine, soit comme mineur en attente de transfert en colonie pénitentiaire. Cependant, les chiffres nationaux sont si conséquents que cela n'a guère d'influence sur les courbes de tendance ${ }^{36}$.

16 La période abordée maintenant s'étend de 1910 à 1945 bien que les graphiques comportent deux «vides" chronologiques. Celui de la Seconde Guerre mondiale d'abord, puisque aucun rapport de fonctionnement n'est édité de 1940 à 1944 . Néanmoins, les statistiques de l'année 1945 existent et, en l'occurrence, apparaissent sur les graphiques. Le second "vide» est celui de l'année 1936. Afin, d'éviter une discontinuité dans les graphiques, susceptible d'en gêner la lecture, le calcul de la moyenne entre le nombre d'entrées de 1935 et de 1937 remplace la section de courbe manquante. 
17 Avant tout examen des mouvements généraux d'entrées des mineurs dans les maisons d'arrêt, de justice et de correction, notons que la période à l'étude est, bien entendu, marquée par les deux conflits mondiaux. La population carcérale s'en trouve forcément marquée à la hausse comme à la baisse. De plus, à la veille de la Première Guerre mondiale, l'activité de l'Administration pénitentiaire est gênée par une restriction des budgets et par le remodelage de la carte pénitentiaire qui consiste principalement en la fermeture de nombreux établissements ${ }^{37}$. Dès la fin du XIX ${ }^{\mathrm{e}}$ siècle et au début du XX $\mathrm{X}^{\mathrm{e}}$ siècle, la population pénale connaît une décrue constante. Ce phénomène est particulièrement visible dans les maisons d'arrêt où l'ensemble de la population, majeurs et mineurs des deux sexes, ne cesse de décroître comme l'illustre la courbe cidessous $^{38}$ :

Graphique 3 : Ensemble de la population détenue, majeurs et mineurs des deux sexes, dans les maisons d'arrêt, de justice et de correction (1880-1909)

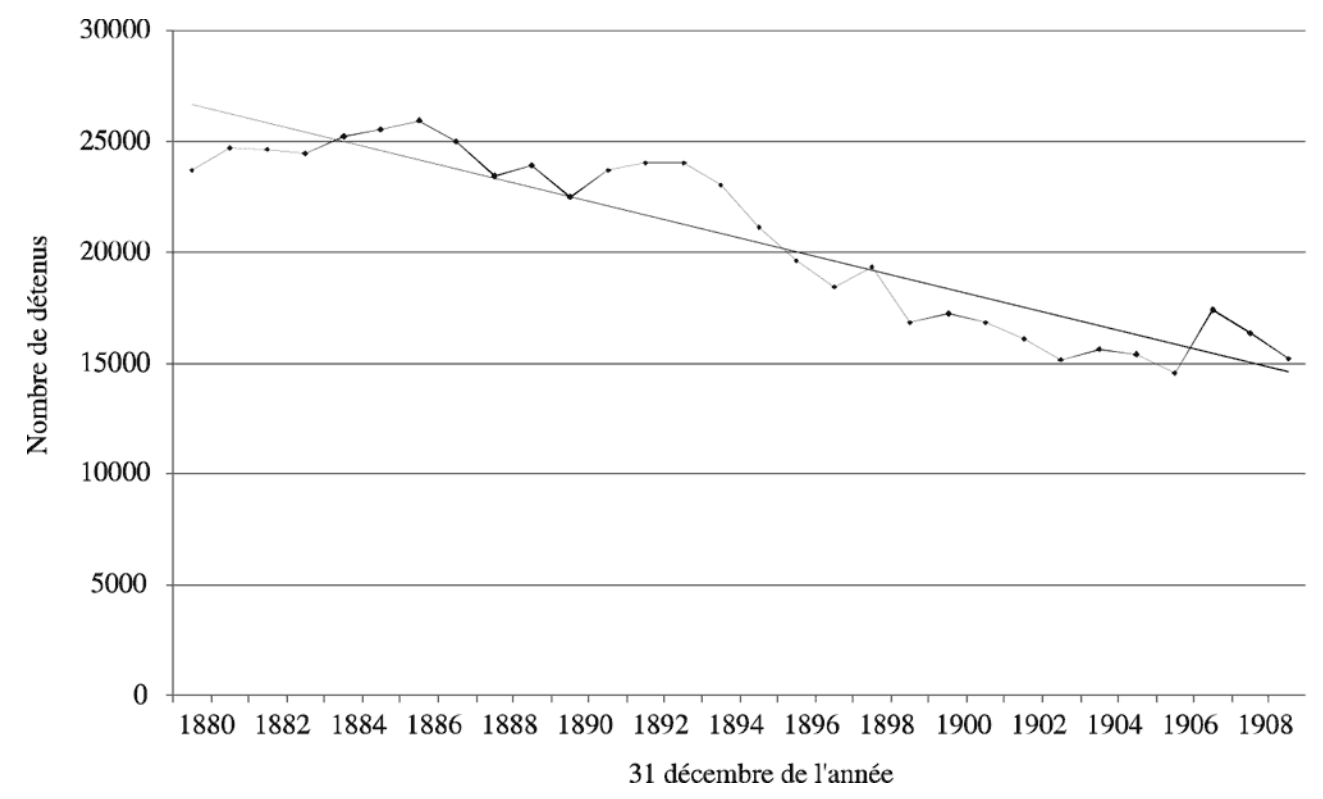

Depuis 1896, le nombre d'individus détenus dans les prisons départementales n'a pas dépassé 20000 unités. Ces rappels effectués, la courbe infra représente l'ensemble des entrées de mineurs pénaux, garçons et filles, dans les maisons d'arrêt, de justice et de correction par année. 
Graphique 4 : Ensemble des mineurs entrés en maison d'arrêt, de justice et de correction au cours de l'année (1910-1945)

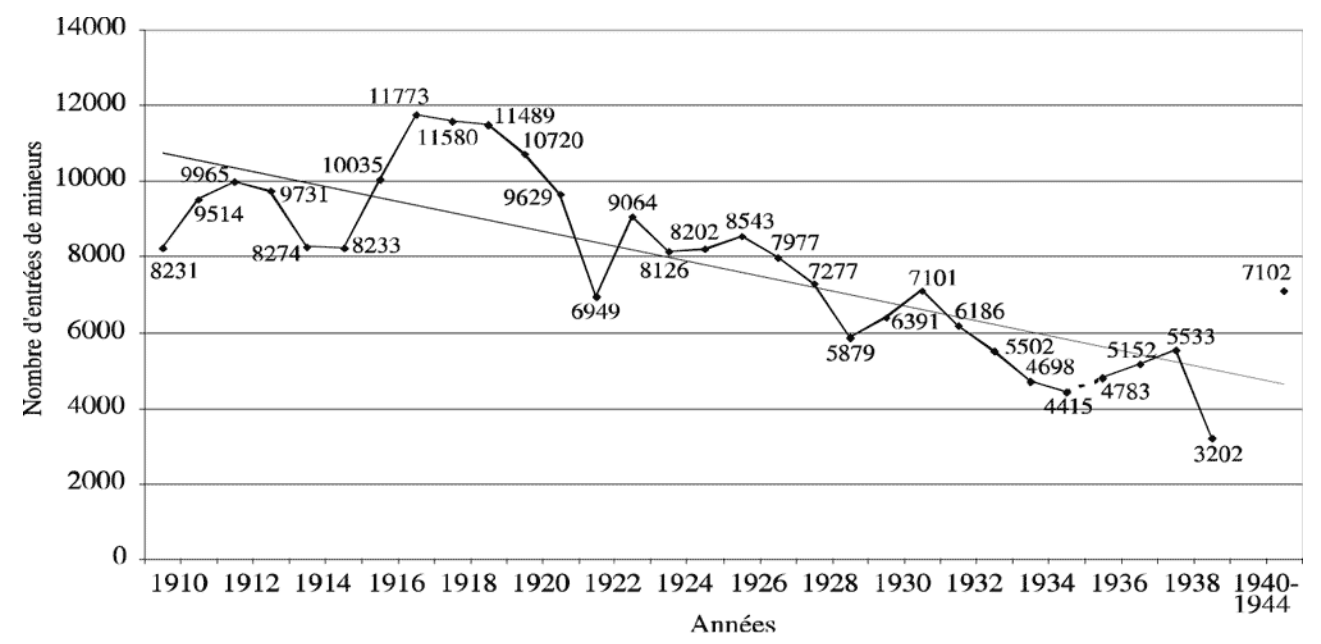

Bien que non linéaire, la tendance générale de la courbe est à la baisse (droite noire). Entre 1910 et 1939, les magistrats semblent de moins en moins recourir à la détention pour les mineurs de justice. Au début de la période, la tendance est à la croissance : pour 8231 entrées en 1910, on en compte 9965 en 1912 soit une augmentation de $21 \%$. À la veille de la Première Guerre mondiale, la courbe descend pendant une courte durée, de 1913 à 1915, avec respectivement 9731 et 8233 passages. Pour cette époque, le nombre d'entrées de mineurs en maisons d'arrêt accuse donc une diminution de 17,4 \% (en 1915 par rapport à 1912) et revient ainsi à la situation de 1910. À cet état, succèdent deux ans de très forte croissance - plus $43 \%$ entre 1915, avec 8233 entrées, et 1917 avec 11773 entrées - suivis par une décroissance, progressive mais importante, qui s'interrompt en 1922. Les 11773 entrées de mineurs en détention en 1917 passent à 6949 entrées en 1922, soit une baisse de $41 \%$. En 1923, la courbe marque un pic qui révèle une augmentation de 211 entrées par rapport à l'année précédente, soit 30,4 \% de hausse, puis elle adopte de nouveau une tendance décroissante jusqu'en 1929. Un nouveau pic est alors visible, moins conséquent, avec une différence de 1222 entrées, soit une augmentation des passages d'enfants en prison de 20,8 \% en 1931 par rapport à 1929. Le dernier mouvement de la courbe accuse une décrue progressive jusqu'en 1935 et marque une diminution des entrées de $37,8 \%$ en quatre ans avant de remonter légèrement jusqu'en 1938. En 1939, la courbe accuse une chute importante de $42,1 \%$ en l'espace d'une seule année. En 1945, le nombre d'entrées des mineurs en détention est au niveau de celui de 1931, à un individu près, soit 7102 passages.

La lecture des variations de cette courbe peut se faire, dans un premier temps, par le biais du double contexte politique et social. Toutefois, l'absence de comparaison possible entre cette courbe et celle établie pour la période précédente, tout comme les comptes faussés par le passage de la majorité pénale à 18 ans en 1906, empêchent de mesurer un phénomène: la répression pénale des apaches, ces bandes urbaines de jeunes qui sévissent dans les villes au début du siècle ${ }^{39}$. Chronologiquement, la décrue de 1913 à 1914 correspond à l'adoption de la loi de 1912 sur les tribunaux pour enfants. Le texte qui instaure la liberté surveillée pour les mineurs, afin de remplacer la détention préventive et de réduire les courtes peines d'emprisonnement, pourrait donc être théoriquement une explication à cette baisse. Cependant, cette loi n'est guère suivie de la création des organes qui auraient permis de l'appliquer ${ }^{40}$ et il faut donc 
chercher ailleurs une justification à cette diminution. Nous sommes à la veille de la Première Guerre mondiale. La mobilisation générale en temps de guerre est requise et implique l'engagement d'un grand nombre de jeunes gens dans l'armée, dont certainement les jeunes Apaches. L'État à besoin de tous les hommes disponibles ce qui le conduit à faire paraître un décret, le 6 août 1914, "autorisant l'engagement des jeunes gens de 17 ans pour la durée de la guerre $»^{41}$ et à recruter au sein-même des établissements pénitentiaires, plusieurs centaines de prévenus ${ }^{42}$, phénomène relevé par le directeur de l'Administration pénitentiaire ${ }^{43}$. Cette disposition contribue indubitablement à alléger la population pénale des maisons d'arrêt et des colonies pénitentiaires ${ }^{44}$.

21 La croissance exponentielle de 1915 à 1917 est tout aussi conjoncturelle. L'incarcération des déserteurs et des rescapés des pelotons d'exécution entraîne l'encombrement des quartiers de droit commun et conduit l'Administration pénitentiaire à vider Fresnes de ses prévenus et la Petite-Roquette de ses mineurs pour en faire des prisons militaires ${ }^{45}$. De plus, la prolongation de la guerre accentue la misère. Une petite délinquance directement liée au conflit mondial se développe alors. Elle touche particulièrement les plus jeunes, filles et garçons. La dislocation des familles amène les enfants seuls à chaparder, à fréquenter les marchés noirs ou à se prostituer :

La fréquence des vols féminins, surtout dans les grands magasins, connaît l'accroissement le plus considérable en raison de la présence sous les drapeaux de nombreux soutiens de famille.

La criminalité infantile épouse la même courbe. Bien des fils de mobilisés se retrouvent livrés à eux-mêmes pendant que la mère travaille ou boit son allocation de femme de mobilisé [...]. On observe aussi une augmentation de la prostitution des mineures $[. . .]^{46}$.

Les prisons pour mineurs se remplissent et, par exemple, "pendant le conflit, les délinquants juvéniles de la Petite-Roquette sont au nombre de cinq cents, alors qu'ils n'étaient que cent cinquante en moyenne auparavant $»^{47}$.

23 Au lendemain de la guerre, la population pénale des mineurs incarcérés en maison d'arrêt reprend sa décrue. Contrairement aux adultes, cette baisse ne peut être imputée aux lois sur la libération conditionnelle et sur la relégation de 1885. En revanche, celle sur le sursis de 1891 et les mineurs placés en liberté surveillée en application de la loi de 1912, contribuent effectivement à la diminution du nombre d'adolescents incarcérés ${ }^{48}$. À ces explications purement législatives s'ajoutent les effets de la guerre : les classes parmi lesquelles se recrutent habituellement les jeunes délinquants sont envoyées au front et ces susceptibles candidats à l'incarcération sont anéantis par les combats. De même, faute de jeunes hommes dans la société civile, le taux de natalité est faible pendant la guerre et, par conséquent, les adolescents sont peu nombreux les années suivantes.

Par la suite, le nombre d'entrées des jeunes délinquants dans les prisons pour courtes peines a une tendance marquée à la décrue. À partir de 1921, le chiffre des 10000 entrées de mineurs en prison n'est plus franchi jusqu'au lendemain de la Seconde Guerre mondiale et atteint même un minima de 4415 en 1935. Le pic de 1923 fait presque figure d'exception mais, à nouveau, il s'explique par l'application d'une nouvelle loi. En effet, si l'article 269 du code pénal stipule que le vagabondage constitue un délit (depuis la grande réforme du 1832), les mineurs ne peuvent plus être condamnés à une peine de prison ${ }^{49}$. Au moment du changement de majorité pénale en 1906, cette disposition, ancienne, s'applique également aux jeunes de 16 à 18 ans qui ne 
peuvent plus être emprisonnés pour vagabondage. La loi du 24 mars 1921 supprime cette restriction et la peine de prison est désormais possible pour cette catégorie. Le pic de 1923 est certainement une démonstration de l'application par les magistrats de cette refonte des articles 269, 270 et 271 du Code pénal. La hausse marquée de 1929 à 1931 correspond à la réouverture par la loi du 22 août 1929 d'un grand nombre de maisons d'arrêt ${ }^{50}$, fermées depuis quatre ans à la suite de la réforme de 1926 sur l'organisation judiciaire. C'est également la période où les garçons sont évacués de la Petite-Roquette pour intégrer Fresnes, plus moderne et plus vaste ${ }^{51}$. Avec davantage de place dans les prisons, il est plus aisé d'y enfermer davantage de détenus ${ }^{52}$. De plus, cette hausse est également, certainement, une conséquence de la réforme de 1927 sur les établissements d'éducation correctionnelle lancée par l'Administration pénitentiaire. Les colonies pénitentiaires deviennent Maison d'Éducation surveillée et ces dernières, c'est désormais de notoriété publique ${ }^{53}$, sont vétustes, mal adaptées et, de fait, délaissées par les magistrats qui préfèrent remettre les enfants à des sociétés de patronages ou à leur famille. Les casquettes des moniteurs-éducateurs, au lieu des képis des gardienssurveillants, ne semblent pas suffire à rassurer les juges sur le sort des enfants dans ces institutions. De plus, il est probable que certains d'entre eux optent, dans le cas de jeunes délinquants récidivistes ou criminels, pour une courte peine d'incarcération plutôt que pour une condamnation à la «maison de correction» ou même en remplacement d'un acquittement pour non-discernement, qui conduit également les mineurs concernés en institution d'éducation correctionnelle. L'usage de la prison est considéré comme un moyen d'effrayer afin de dissuader à la perpétration d'un délit. Cette hypothèse trouve un écho dans la tendance qu'adopte la courbe vers 1935-1936. Le nombre de mineurs qui passe par la maison d'arrêt est alors à nouveau à la hausse, et correspond aux vigoureuses campagnes contre les « bagnes d'enfants » des années 1936 et 1937. Les mêmes causes ont les mêmes conséquences. Les propositions d'explications de la courbe de l'incarcération des mineurs, de la déchéance des colonies pénitentiaires à l'utilisation de la prison "coup d'arrêt ", sont confortées par l'examen de quelques données sur les entrées annuelles de garçons dans les établissements publics, en baisse régulière : 1738 entrées pour l'année 1920 (dont 967 garçons venus directement des prisons départementales), 1302 entrées en 1925 (dont 493 provenant de maisons d'arrêt), 1004 en 1931 (dont 514 venant des maisons d'arrêt), 876 en 1935 (dont 466 venus de prison), 796 en 1939 (dont seulement 79 venant de prison). À cette tendance s'ajoute une amélioration du régime de détention des mineurs dans les maisons d'arrêt, susceptible de donner bonne conscience aux juges ${ }^{54}$.

La brusque décrue du nombre d'entrées de mineurs en maisons d'arrêt en 1939 par rapport à 1938 (moins 42,1\%) s'explique de nouveau par le début de la guerre et le lot de réquisitions et d'incorporations plus ou moins forcées des jeunes délinquants d'habitude qui l'accompagne. Ce qui se passe entre 1940 et 1944 dans les prisons françaises, combien d'adolescents y sont enfermés, il est difficile de le savoir et la courbe n'est ici d'aucun secours puisque les rapports statistiques manquent. Les données disponibles permettent tout de même de constater que les jeunes détenus passés par une maison d'arrêt en 1945 sont bien plus nombreux qu'avant guerre. Avec 7102 entrées, l'importance de la détention des mineurs est à un niveau jamais atteint depuis $1931^{55}$. Parmi ces jeunes gens en prison, les mêmes petits délinquants issus de la conjoncture liée au conflit sont présents ${ }^{56}$ mais certains sont de jeunes collaborateurs et autres auteurs d'« atteinte à la sûreté de l'État $\|^{57}$. 
Au delà des considérations d'ordre général liées au contexte social et politique et aux grands changements législatifs ou de l'Administration pénitentiaire, il convient d'examiner les différences entre l'incarcération des garçons d'une part et celle des filles d'autre part.

Graphique 5 : Entrées des filles et des garçons en maison d'arrêt, de justice et de correction au cours de l'année (1910-1945)

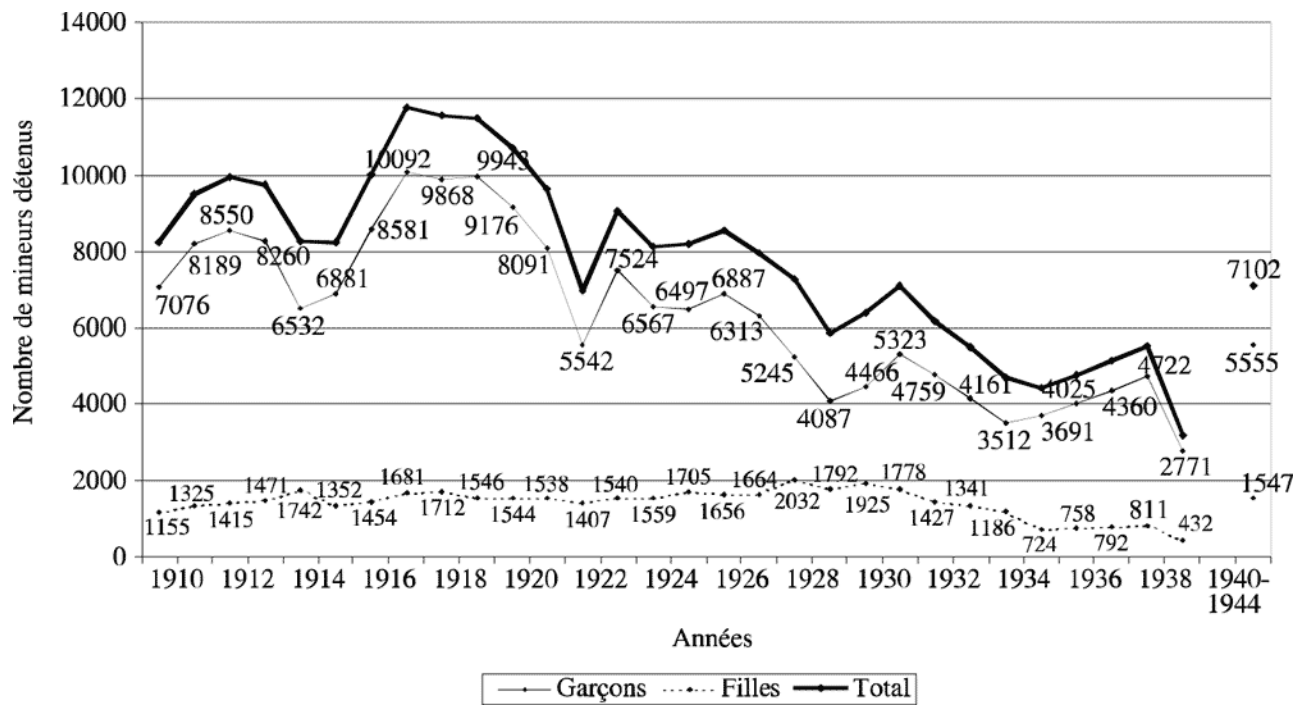

Plus encore que pour la période étudiée précédemment, la courbe des entrées des garçons suit précisément celle établie pour l'ensemble de la population des jeunes détenus. La courbe qui représente les entrées annuelles des filles semble à peu près constante, avec une légère tendance à la baisse, et n'a visiblement aucune influence sensible, à aucun moment, sur la courbe générale. Le phénomène est aisé à comprendre. Cette période est marquée par les faits de guerre, or c'est la population masculine, la plus nombreuse, qui est la plus concernée par la mobilisation et par les arrestations. Les variations de la courbe générale sont donc représentatives des facteurs conjoncturels qui touchent les garçons. Pourtant, à l'examen de l'histogramme infra, les entrées en maisons d'arrêt des filles représentent un minimum de $15 \%$ des entrées pour l'ensemble des jeunes détenus et atteignent un maximum de $30 \%$ en 1929. Sur cette période, le nombre d'entrées des filles dans les prisons départementales sur l'ensemble des jeunes détenus est, en moyenne, supérieur à celui de la période précédente. Le seul mouvement qui rompt la constance de la courbe consacrée aux filles, est le pic de 1945. Les fluctuations de 1910 à 1938 semblent toutes d'intensité réduite. Cependant, une tendance décroissante s'affirme à partir de 1930. Ramenée à une autre échelle, la fluctuation de la courbe des passages des mineures en prison apparaît plus accentuée. La baisse du recours à l'incarcération à partir de 1930 devient plus visible: 
Histogramme 2 : Pourcentage des filles détenues en maison d'arrêt, de justice et de correction, par rapport à l'ensemble des jeunes détenus (1910-1945)

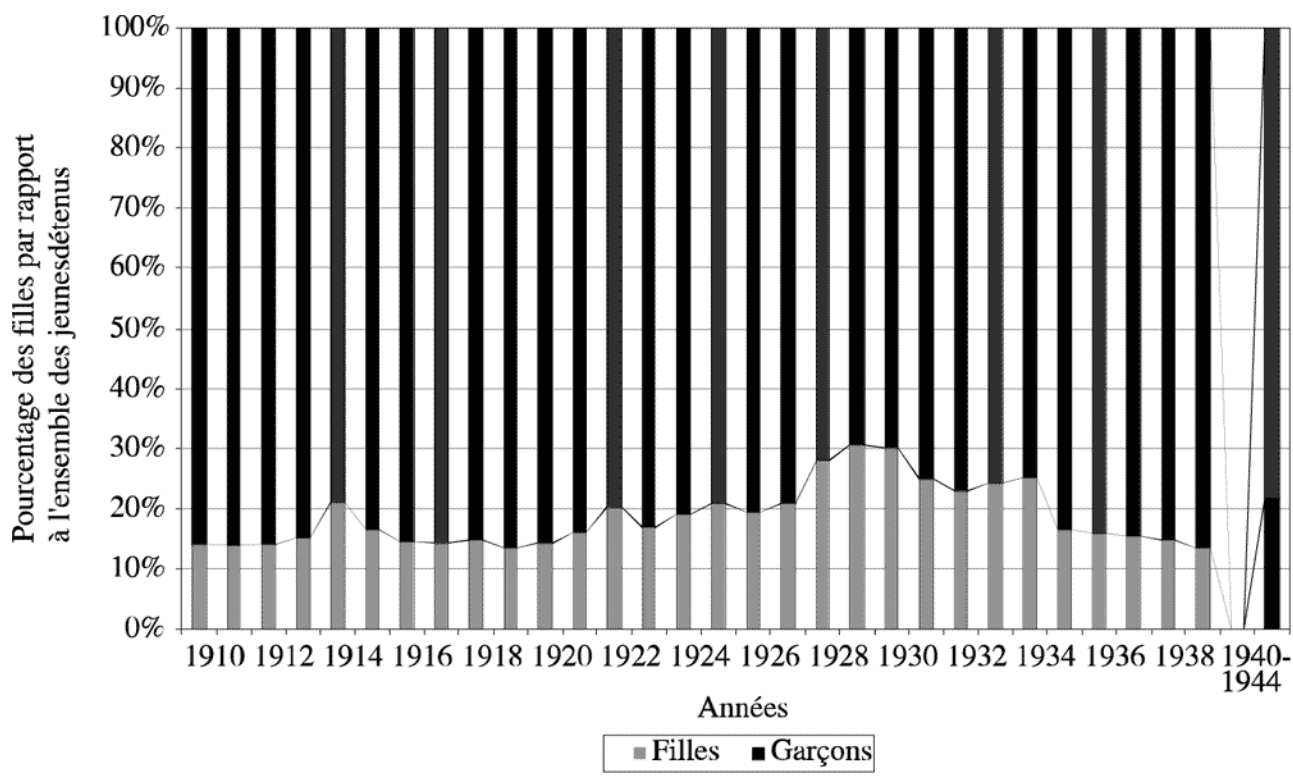

Finalement, des tendances équivalentes à celles des garçons se dégagent de cette courbe consacrée aux filles. Ainsi, l'après Première Guerre mondiale, en 1917 et 1918, est une période de recrudescence du nombre d'entrées en prison. Les mêmes faits de collaboration peuvent être mis au crédit de cette augmentation du nombre de jeunes détenues ${ }^{58}$. De même, les augmentations du nombre de jeunes filles dans les maisons d'arrêt en 1928-1930 et 1936-1938 trouvent une justification dans le discrédit dont pâtissent l'ensemble des Maisons d'Éducation surveillée dont les Écoles de préservation, réservées à la gent féminine, ne sont pas exclues. De plus, la loi de 1935, qui établit que la correction paternelle ne se subira plus en prison, ne semble pas vraiment influencer la courbe des demoiselles détenues en maisons d'arrêt. Acontrario, certaines directions prises par cette dernière sont à l'inverse de celle des garçons. Par exemple, en 1914, alors que les garçons, mobilisés, sont sortis des prisons, les filles, acculées à une délinquance de survie, y entrent en plus grand nombre. De même, le pic de 1928, visible sur la courbe féminine, ne l'est pas sur la courbe masculine. En effet, la Petite-Roquette, vidée des garçons, est désormais réservée aux femmes, dont très probablement un certain nombre de jeunes filles ${ }^{59}$.

\section{II - Quelle situation légale pour les jeunes détenus des maisons d'arrêt ?}

Dans les prisons départementales, un mineur ou une mineure détenue peut appartenir à l'une des trois situations légales existantes, elles-mêmes sous-divisées en deux ou trois catégories. Elles sont distinguées et explicitées, dans le tableau suivant : 
Document 2 : Les différentes situations légales des mineurs détenus en maison d'arrêt répertoriées dans les rapports de Statistiques pénitentiaires

\begin{tabular}{|l||l|l||l|}
\hline $\begin{array}{l}\text { Prévenu(e)s } \\
\text { accusé(e)s }\end{array}$ & $\begin{array}{l}\text { Soit en attente } \\
\text { d'être jugés }\end{array}$ & $\begin{array}{l}\text { Les prévenu(e)s sont suspecté(e)s de } \\
\text { délit, les accusé(e)s de crimes }\end{array}$ & $\begin{array}{l}\text { Catégories mêlées dans les } \\
\text { tableaux de 1880 à 1909 - } \\
\text { Séparées dans les tableaux } \\
\text { de 1910-1945 }\end{array}$ \\
\cline { 2 - 4 } & $\begin{array}{l}\text { Soit en appel ou } \\
\text { en pourvoi }\end{array}$ & $\begin{array}{l}\text { Le jugement a été contesté, attente } \\
\text { d'une éventuelle révision du procès }\end{array}$ & \\
\hline
\end{tabular}

\begin{tabular}{|c|c|c|c|c|}
\hline \multirow[t]{3}{*}{$\begin{array}{l}\text { Conda- } \\
\text { mné(e)s }\end{array}$} & \multirow{2}{*}{\begin{tabular}{|l|} 
Soit condamné(e)s \\
à moins \\
de $\quad 6 \quad$ mois \\
d'emprisonnement
\end{tabular}} & \multicolumn{2}{|c|}{$\begin{array}{l}\text { Condamné(e)s à purger en maison d'arrêt une peine } \\
\text { privative de liberté inférieure ou égale à six mois }\end{array}$} & \multirow{3}{*}{$\begin{array}{l}\text { Catégories } \\
\text { compta- } \\
\text { bilisées } \\
\text { séparément } \\
\text { dans les } \\
\text { tableaux } \\
\text { statistiques }\end{array}$} \\
\hline & & $\mid \begin{array}{l}\text { Soit mineurs de } 16 \text { ans «condamnés à un } \\
\text { emprisonnement de plus de six mois et qui } \\
\text { n'excède pas deux ans » et qui, en vertu de la loi } \\
\text { du } 12 \text { août } 1850 \text {, doivent purger leur peine dans } \\
\text { une institution d'éducation correctionnelle }{ }^{60}\end{array}$ & \begin{tabular}{|l} 
Non différen \\
ciées dans les \\
tableaux \\
statistiques
\end{tabular} & \\
\hline & & $\begin{array}{l}\text { Soit mineur(e)s envoyé(e)s en état de dépôt ou } \\
\text { d'arrêt dans les prisons départementales, } \\
\text { reconnu(e)s comme ayant agi sans discernement } \\
\text { mais non remis(es) aux parents, conduit(e)s dans } \\
\text { une colonie pénitentiaire pour y être enfermé(e)s } \\
\text { en vertu de l'article } 66 \text { du code pénal }\end{array}$ & & \\
\hline
\end{tabular}

\begin{tabular}{|l|l|l|l|}
\hline Interné(es) & $\begin{array}{l}\text { Soit par voie de } \\
\text { correction } \\
\text { paternelle }\end{array}$ & (Catégorie présente jusqu'en 1935) & $\begin{array}{l}\text { Catégories } \\
\text { différenciées dans les } \\
\text { tableaux statistiques }\end{array}$ \\
\cline { 2 - 4 } & $\begin{array}{l}\text { Soit venant de } \\
\text { l'assistance } \\
\text { publique }\end{array}$ & $\begin{array}{l}\text { (Catégorie présente à partir de 1910). } \\
\text { sureur(e)s relevant de la loi du 28 juin 1904 } \\
\text { publique difficiles ou vicieux }\end{array}$ & \\
\hline
\end{tabular}

Il s'agit ici d'effectuer une étude comparative de ces différentes situations des jeunes détenus en maison d'arrêt, de justice et de correction afin d'y déterminer la place respective des prévenu(e)s, des condamné(e)s et des interné(e)s. Cet examen permet quelques interprétations des pratiques des magistrats et de vérifier dans quelle mesure les textes législatifs sont, ou non, pris en compte par ces derniers. 


\section{Vers la généralisation de l'incarcération préventive de 1880 à} 1909

Le graphique suivant affiche une courbe pour chaque grand groupe de mineurs en détention : les prévenus et accusés des deux sexes; les condamnés à moins de six mois et en attente de transfert, garçons et filles; les mineurs de la correction paternelle.

Graphique 6 : Ensemble des prévenus et accusés, des condamnés et des internés mineurs incarcérés en maison d'arrêt, de justice et de correction au 31 décembre de l'année (1880-1909)

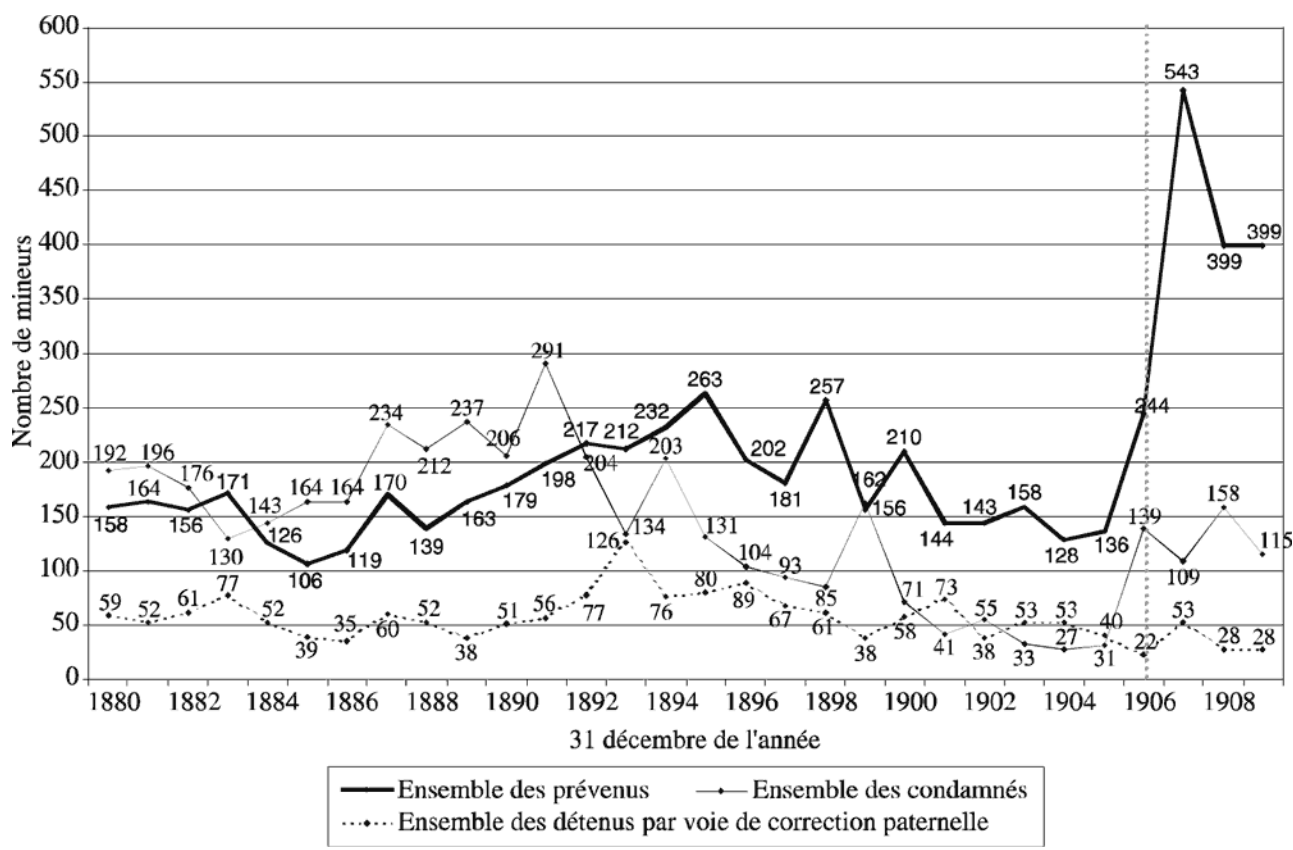

Au début de la période, de 1880 à 1882, les jeunes condamnés à moins de six mois de détention ou en transfert présents au 31 décembre dans les maisons d'arrêt, sont légèrement plus nombreux que les jeunes prévenus et accusés : 34 condamnés de plus que de prévenus en 1880, 32 en 1882 et 20 en 1882. Le temps d'une année, la tendance s'inverse et les jeunes prévenus et accusés sont 41 de plus que leurs codétenus condamnés avant que, de nouveau, ces derniers soient davantage représentés que les enfants en attente de jugement. Jusqu'en 1892, c'est cette tendance qui reste la règle. L'écart le plus important est alors de 93 condamnés de plus que de prévenus et accusés en 1991. L'écart le moins important est de 27 individus l'année précédente, en 1890. Puis, la courbe des jeunes prévenus et accusés détenus au 31 décembre dépasse de nouveau celle des jeunes condamnés en 1892 et reste au-dessus jusqu'à la fin de la période. Les écarts entre les deux types de situations légales sont, dans l'ensemble, plus conséquents que précédemment. La différence maximum est celle de 1898 avec 172 détenus et accusés incarcérés au 31 décembre de plus que de condamnés. En 1899 cependant, il est à noter que la courbe des prévenus et accusés se rapproche de celle des condamnés, avec respectivement 162 prévenus et accusés et 156 condamnés ou en attente de transfert, soit une différence de 6 enfants seulement. La courbe des prévenus et accusés reprend alors son indépendance et demeure bien au-dessus de celle des condamnés. Après 1906, avec l'intégration dans les comptes des adolescents de 16-18 ans, l'écart entre les prévenus et accusés et les condamnés se creuse. Pour 1907, au 31 décembre, on dénombre 434 mineurs condamnés de plus que de prévenus et accusés. 
Jusqu'en 1900, les enfants détenus par voie de correction paternelle sont les moins nombreux. Avant 1892, ils ne dépassent pas la centaine d'individus alors que sont incarcérés un minimum de 106 prévenus et un minimum de 130 condamnés. Fin décembre 1893, le nombre d'enfants de la correction paternelle atteint son maximum avec 126 unités, ils sont alors presque aussi nombreux que les jeunes condamnés ou en attente de transfert (134). Cependant, la courbe reprend aussitôt sa tendance à la baisse. De nouveau, les jeunes victimes punies par leur père n'atteignent plus la centaine et la prise en compte des 16-18 ans ne modifie rien. De fait, de 1900 à 1905, la courbe des enfants de la correction opère un chassé-croisé avec celle des enfants condamnés pendant que celle des prévenus et accusés reste largement au-dessus. Ils sont 13 jeunes de la correction de moins que les jeunes condamnés en 1900, puis 32 de plus en 1901, puis de nouveau 17 de moins en 1902, puis respectivement 10, 26 et 9 de plus pour les années 1903, 1904 et 1905 avant d'être définitivement moins nombreux que les condamnés.

Malgré cette comparaison quantitative, le nombre d'enfants de la correction paternelle ne dépend en rien du nombre de jeunes condamnés et de jeunes prévenus et accusés. Les variations de la courbe des enfants de la paternelle sont totalement indépendantes puisque le système de la correction paternelle dépend du civil et non du pénal. Les chiffres en eux-mêmes ne permettent d'ailleurs pas de se faire une idée précise de l'importance du recours à l'incarcération en maison d'arrêt pour ces enfants. Si elles offrent quelques idées approximatives, les statistiques fournies par les rapports de Statistiques pénitentiaires ne donnent que l'état de la population pénitentiaire à la fin de l'année civile. La plupart des jeunes concernés par la correction, détenus seulement pour quelques semaines, six mois au maximum, échappent donc aux décomptes. Bernard Schnapper, qui s'appuie sur LeCompte général de l'administration de la justice civile, analyse la période dont il est question comme le moment "d'hésitation, de repentir " ${ }^{61}$ avant l'amorce, en 1905, "d'une longue baisse qui annonce la période finale ${ }^{62}$. De plus, l'historien insiste sur les différences de pratiques entre le département de la Seine et ceux de la province. Ces constats, ne se lisent guère sur la courbe nationale composée à partir des rapports de l'Administration pénitentiaire pour les raisons précédemment évoquées. Tout juste est-il possible de percevoir dans la tendance descendante de la courbe aux débuts des années 1890, l'impact des débats contre la correction paternelle et les débuts de la désuétude de cette pratique. Néanmoins, cette courbe permet de percevoir la différence quantitative de ces " corrigés " par rapport aux autres mineurs détenus en fin d'année à la même époque, prévenus, accusés et condamnés. Il serait cependant vain, de tenter de faire dire autre chose à ces chiffres. De 1880 à 1892, la courbe du nombre d'enfants de la correction paternelle présente des similitudes dans ces mouvements, à un niveau différent, avec celle des jeunes prévenus et accusés. La ressemblance disparait à partir de 1893. Il est probable, que durant ces quelques années, la correction fonctionne comme un substitut de la répression. Les conclusions pour l'analyse de cette courbe ne peuvent aller au delà de ce constat.

En ce qui concerne les courbes formées par les données relatives aux prévenus et aux condamnés, les comparaisons ne sont pas en inadéquation. Il n'est pas aisé d'expliquer la différence, selon les périodes, entre le nombre de prévenus et accusés et celui de condamnés. Certains phénomènes peuvent toutefois être relevés. L'histogramme infra 
met en évidence les différences quantitatives entre les deux catégories de jeunes incarcérés :

Histogramme 3 : Ensemble des prévenus et accusés et des condamnés détenus dans les maisons d'arrêt, de justice et de correction au 31 décembre de l'année (1880-1909)

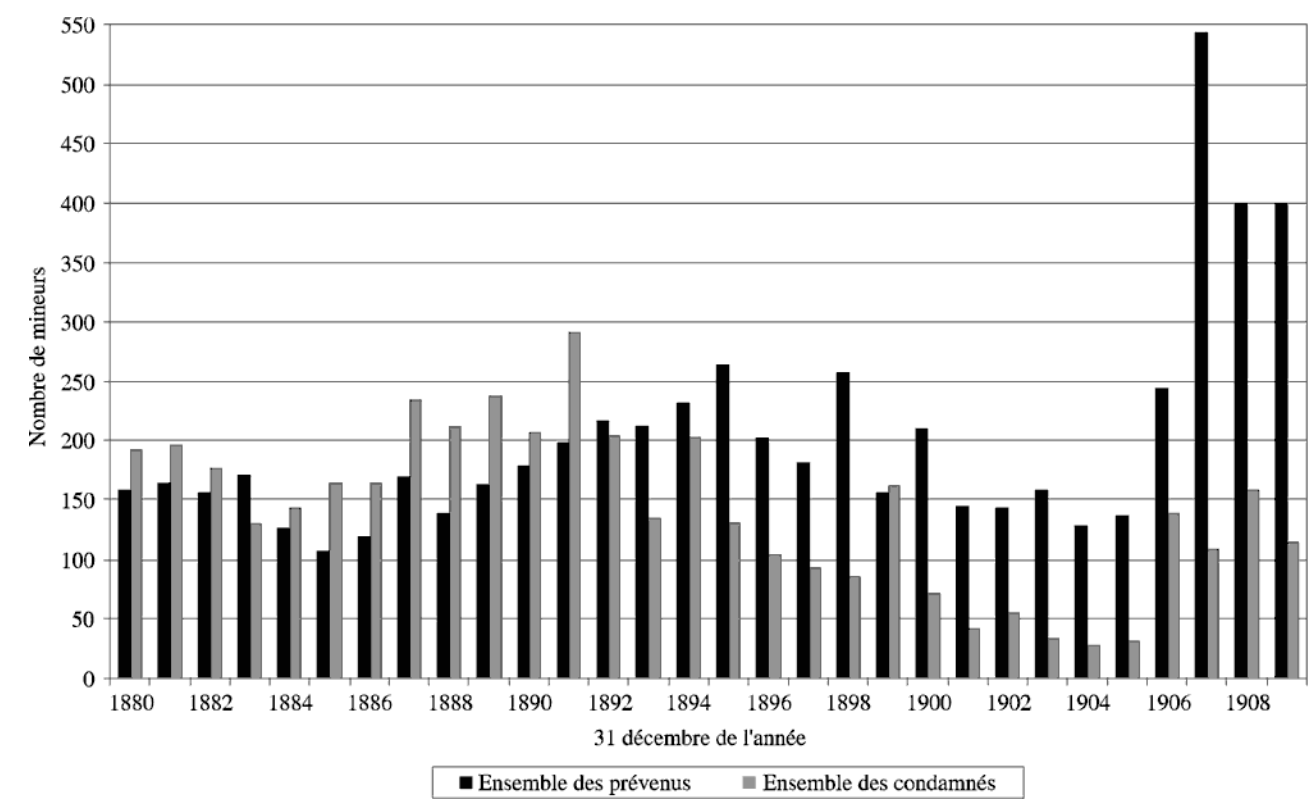

Parfois, les différents graphiques reflètent parfaitement les changements de législation. D'autres fois, ils mettent en évidence les non-applications d'une loi ou d'une mesure judiciaire. En 1893, par exemple, les tendances s'inversent et les jeunes condamnés, jusqu'alors plus nombreux que les jeunes prévenus, sont désormais bien inférieurs en nombre. C'est la loi du 15 novembre $1892^{63}$ qui explique ce phénomène. En effet, celle-ci impute la détention préventive sur la durée des peines prononcées. Ce temps de détention pendant l'attente du jugement définitif s'ajoute donc à la condamnation. De fait, avant 1892, dans un compte en "stock", de nombreux condamnés à de courtes peines sont comptabilisés. Après la loi de 1892, ils sont forcement moins nombreux puisque certains d'entre eux ont été libérés, ayant fait leur peine sur le temps de la préventive. On compte donc forcement plus de jeunes condamnés à de courtes peines en décembre 1891 qu'en décembre 1893 , ce que reflètent parfaitement le graphique $n^{\circ} 6$ et l'histogramme $n^{\circ} 3$. Cependant, cette loi n'est pas toujours bien comprise et ne profite pas à tous les enfants détenus préventivement. En effet, si un mineur, après quelques temps de détention en préventive dans une maison d'arrêt, est reconnu comme ayant agi sans discernement mais conduit en colonie pénitentiaire pour un temps déterminé, la détention préventive n'est pas imputée sur le temps à subir dans l'institution d'éducation correctionnelle. En témoignent les extraits de l'échange épistolaire reproduit ci-dessous :

[Lettre du président du conseil à M. le ministre de la Justice, 19 octobre 1907]

Le jeune G. (Léonard), âgé de 15 ans, a été traduit, pour abus de confiance et vols devant le tribunal d'Amiens qui, par jugement du 4 octobre 1907 [...] l'a acquitté comme ayant agi sans discernement, mais a ordonné qu'il serait conduit dans une colonie pénitentiaire pendant trois mois par application de l'art. $66 \mathrm{du}$ code pénal. G. ayant été placé sous mandat de dépôt et écroué le 26 juillet 1907, aura subi trois mois de détention le 26 octobre courant. 
Je vous serais obligé de vouloir bien me faire connaître si, en présence des termes du jugement qui prononce l'envoi en correction non pas jusqu'à un âge mais pendant un laps de temps déterminé, il vous paraît qu'il y ait lieu de faire bénéficier le mineur des dispositions de la loi du 15 novembre 1892 visant l'imputation de la détention préventive sur toute peine privative de la liberté.

[Réponse du ministre de la Justice, 29 octobre 1907] imputation de détention préventive pour ce motif péremptoire qu'il n'y a pas de peine (art. $66 \mathrm{CP}$ ) prononcée, et que l'imputation ne peut se faire que sur les peines [...] ${ }^{64}$.

De plus, désormais, la condamnation à l'envoi en correction, lorsqu'elle est inférieure à six mois, est exécutée en maison d'arrêt... nouveau paradoxe de l'article 66.

À l'inverse du respect de la loi du 15 novembre 1892, les graphiques prouvent la faible application de la loi du 19 avril 1898. Celle-ci préconise que «le juge d'instruction commis pourra, en tout état de cause ordonner [...] que la garde de l'enfant soit provisoirement confiée, jusqu'à ce qu'il soit intervenu une décision définitive, à un parent, à une personne ou à une institution charitable qu'il désignera, ou enfin à l'Assistance publique $»^{65}$. Cette mesure doit donc permettre aux magistrats de réduire le nombre de mineurs placés en préventive dans les maisons d'arrêt. Auparavant, les juges disposaient uniquement de l'incarcération préventive comme moyen de s'assurer de la présence du jeune à l'audience, aussi y font-ils fréquemment appel. Les nombres de jeunes condamnés et de jeunes prévenus similaires en 1899, contrairement aux six années précédentes où les prévenus sont largement majoritaires, laissent penser que la loi est appliquée. Cependant, dès l'année suivante, les jeunes prévenus et accusés sont à nouveau bien plus nombreux à être détenus que les jeunes condamnés (139 individus de plus au 31 décembre). La loi de 1898 ne modifie donc pas réellement la situation antérieure. En effet, les institutions «charitables» en question, restent peu nombreuses et surpeuplées ${ }^{66}$. Les alternatives à la remise aux parents et à l'emprisonnement sont fort rares à l'époque.

Si la courbe des jeunes prévenus et accusés n'appelle pas de commentaire supplémentaire, celle des jeunes condamnés mérite une étude plus détaillée. En effet, les mineurs condamnés sont répartis en deux catégories : les condamnés à moins de six mois d'emprisonnement et ceux qui, condamnés à plus de six mois mais moins de deux ans, sont dans l'attente de leur transfert dans une colonie pénitentiaire. La part de chacune de ces catégories de condamnés est représentée dans le graphique ci-dessous. 
Graphique 7 : Jeunes condamnés à moins de six mois d'emprisonnnement et jeunes condamnés attendant leur transfèrement, incarcérés en maison d'arrêt, de justice et de correction au 31 décembre de l'année (1880-1909)

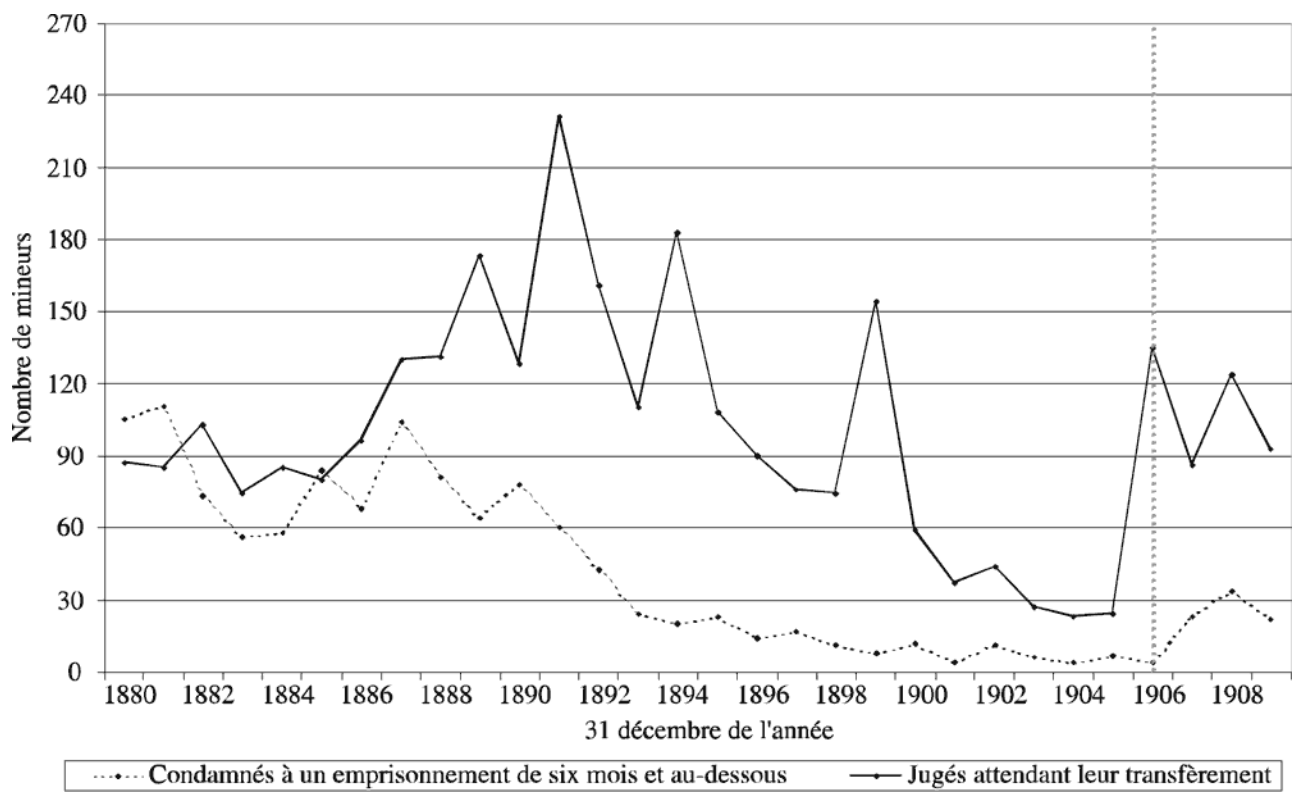

Ce graphique confirme la nette tendance à la baisse, après 1892, du nombre de jeunes condamnés, détenus au 31 décembre, à des peines d'emprisonnement inférieures à six mois. Outre ce phénomène déjà explicité, il faut souligner le statut bien particulier des jeunes en attente de transfert. En effet, législativement, les mineurs condamnés à moins de six mois d'emprisonnement sont à leur place dans les maisons d'arrêt, de justice et de correction même s'il arrive exceptionnellement qu'ils purgent leur peine dans les colonies pénitentiaires. Habituellement, la tendance est davantage à la prolongation d'une incarcération plus ou moins licite. En effet, d'après la loi de 1850, les jeunes condamnés à plus de six mois d'emprisonnement doivent être retenus dans les institutions d'éducation correctionnelle. Leur présence dans les prisons départementales ne saurait donc s'éterniser au risque de devenir illégale. Pourtant, d'après le graphique, ils sont, pendant la plus grande partie de la période, largement supérieurs en nombre aux condamnés à de courtes peines. C'est que les institutions de correction sont régulièrement combles et que les services de transfèrements sont souvent inopérants ou aléatoires. D'ailleurs, le tracé perturbé de la courbe des jeunes en attente de transfert reflète bien ces dysfonctionnements et elle serait certainement encore davantage chaotique pour un décompte en "flux " plutôt qu'en "stock", comme c'est le cas ici. La situation est plus ambivalente encore pour les jeunes acquittés comme ayant agi sans discernement. En effet, les adolescents de cette catégorie, non coupables, ne devraient pas être en prison, or, une grande partie des enfants des colonies pénitentiaires viennent de prisons départementales : la détention préventive est extrêmement courante avant le placement en institution d'éducation correctionnelle ou la remise aux parents. De fait, la détention préventive est quasi systématique après une arrestation. Des mineurs acquittés lors de leurs jugements ont généralement subi quelques temps d'incarcération en maison d'arrêt. Les registres des jugements correctionnels regorgent de cas relevant de ce phénomène. Par exemple, Aimé B. et Auguste $\mathrm{F}$., âgés de 10 et 7 ans ont été remis à leurs parents après au moins onze jours de détention préventive ${ }^{67}$ (cf. document 3 infra). 


\section{L'irréversible hégémonie de la détention préventive de la première moitié du XXe siècle}

41 De 1910 à 1945, l'unité de compte n'est plus le mineur détenu au 31 décembre de l'année mais le nombre d'entrées, au cours de l'année. Cette nouvelle mesure implique des chiffres conséquents. Or, le nombre d'entrées de mineurs détenus par voie de correction paternelle et en vertu de la loi du 28 juin 1904 est bien moindre que celui des condamnés et des prévenus et accusés. Il est donc impossible de faire figurer sur le même graphique les courbes correspondant aux trois situations légales des détenus mineurs. Un graphique semi-logarithmique peut, certes, obvier à cette difficulté mais il a le désavantage de moins bien rendre compte des mouvements de chacune des courbes. De plus, et surtout, la détention par voie de correction paternelle relève de procédures civiles et ne peut être comparée aux procédures pénales dont relèvent les prévenus, accusés et condamnés. Aussi, le choix de traiter, d'une part, des prévenus et des condamnés et, d'autre part, des internés s'impose-t-il. Les courbes infra sont, par conséquent, celles du nombre d'entrées de prévenus de moins de 18 ans au cours de l'année et celles du nombre d'entrées de condamnés de moins de 18 ans au cours de l'année.

Document 3 : Extrait d'un registre de jugements correctionnels de la Vienne - $1898^{68}$

\begin{tabular}{|c|c|}
\hline $\begin{array}{l}\mathbf{N}^{\circ} \text { DU JUGEMENT } 21 \\
\mathbf{N}^{\circ} \text { DU PARQUET } 64\end{array}$ & $\begin{array}{l}\text { Audience du } \\
22 \text { mars } 1898\end{array}$ \\
\hline 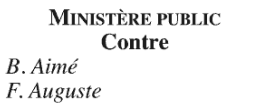 & $\begin{array}{l}\text { Entre Monsieur le procureur de la République près du } \\
\text { Tribunal de police correctionnelle de Châtellerault, } \\
\text { poursuivant d'office; } \\
\text { Et: B. Aimé - } 10 \text { ans, né à Inorandes le } 11 \text { mars } 1887 \text { - }\end{array}$ \\
\hline $\begin{array}{l}\quad \text { Nature du délit } \\
\text { Contravention à la police } \\
\text { des chemins de fer } \\
\text { Art. } 66 \text { du CP et } 61 \text { de } \\
\text { l'ordo. du } 15 \text { nov. } 1846\end{array}$ & $\begin{array}{l}\text { habite chez ses parents à Châtellerault } \\
\text { F. Auguste - } 7 \text { ans - né à Châtellerault le } 3 \text { juin } 1890 \text { - } \\
\text { habite chez ses parents } \\
\text { Prévenu de contravention à la police des chemins de fer }\end{array}$ \\
\hline $\begin{array}{l}\quad \text { Poursuite } \\
\text { Flagrant délit // } \\
\text { Directe } \\
\text { Information // }\end{array}$ & $\begin{array}{l}\text { Présent aux débats non détenu } \\
\text { À l'appel de la cause M. Vesco } \\
\text { Procureur de la République, a exposé que le surnommé } \\
\text { avait été invité }\end{array}$ \\
\hline $\begin{array}{l}\quad \text { Condamnation } \\
\text { Emprisonnement } / / \\
\text { Amende acquittés et remis } \\
\text { à leurs parents }\end{array}$ & $\begin{array}{l}\text { À comparaître par devant le Tribunal, à la présente } \\
\text { audience, pour se défendre en raison de la prévention } \\
\text { ci-dessus indiquée. }\end{array}$ \\
\hline $\begin{array}{l}\text { Le // } \\
\text { Délivré un extrait pour la } \\
\text { prison à M. le procureur } \\
\text { de la République }\end{array}$ & $\begin{array}{l}\text { Puis il a été procédé à l'audition des témoins sous la foi } \\
\text { du serment. } \\
\text { Le prévenu a été interrogé } \\
\text { M. Vesco } \\
\text { Procureur de la République, à résumé l'affaire et } \\
\text { requis: l'application des art. 61 de l'ordonnance du } \\
15 \text { novembre } 1846 \text { et } 66 \text { du CP } \\
\mathbf{M}^{\mathbf{c}}\end{array}$ \\
\hline $\begin{array}{l}\text { ment; } \\
\text { Duplicata pour casier } \\
\text { administratif; } \\
\text { A M. le Procureur de la } \\
\text { République }\end{array}$ & $\begin{array}{l}\text { Sur quoi: } \\
\text { Ouï le prévenu en son interrogatoire et le Ministère } \\
\text { public en ses réquisitions: } \\
\text { Ouï } \mathbf{M}^{\mathbf{c}} \\
\text { Attendu ont jeté des pierres et des boules de neige sur } \\
\text { train. Cassé un carreau } \\
\Rightarrow \text { remis à leurs parents car moins de } 16 \text { ans donc art. } 66 \text {. } \\
\text { Pères ont frais liquidé }\end{array}$ \\
\hline
\end{tabular}


Graphique 8 : Ensemble des mineurs prévenus et condamnés entrés en maison d'arrêt, de justice et de correction pendant l'année (1910-1945)

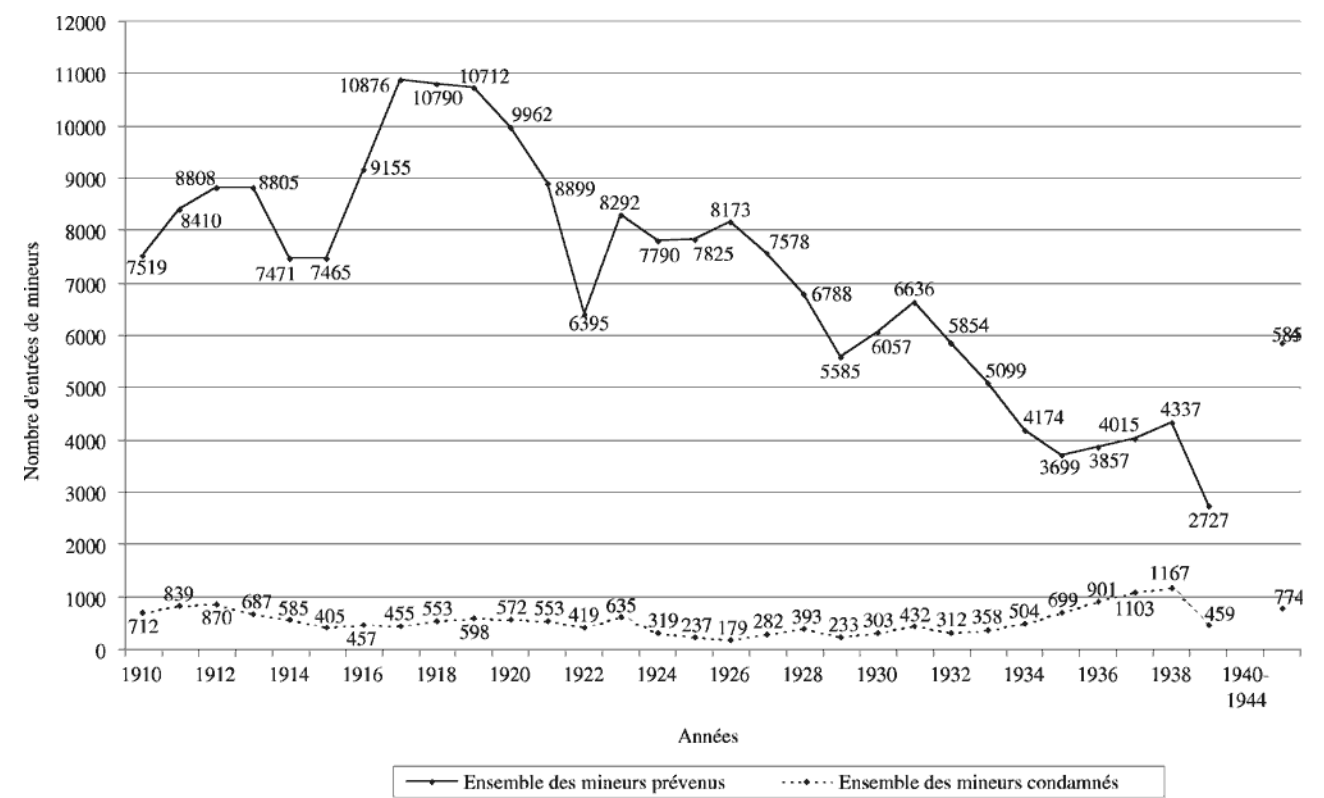

Un constat principal s'impose : le nombre d'entrées des jeunes condamnés est bien plus faible que celui des prévenus. Les jeunes condamnés à moins de six mois d'emprisonnement, ou en attente de transfert, enregistrés au cours d'une année ne dépassent guère les 870 unités ${ }^{69}$ sauf pour les années 1937 et 1938 qui comptent respectivement 1103 et 1167 entrées sur douze mois. Quant à la courbe qui représente le nombre d'entrées des prévenus, accusés et jeunes en pourvoi ou en appel, elle accuse les mêmes variations que celle du graphique $n^{\circ} 4$ consacré à l'ensemble des mineurs détenus de 1910 à 1945. C'est que le nombre d'entrées des condamnés est trop inférieur à celui des jeunes prévenus et accusés pour influer sur la courbe générale. Il apparaît donc que les explications données dans la partie précédente, des états de guerre aux réformes des colonies pénitentiaires en passant par les changements législatifs, s'appliquent essentiellement à la situation des jeunes en attente de jugement ou en appel.

Les rapports de Statistique pénitentiaire, de 1910 à 1945, contrairement à la période précédente, différencient les adolescents prévenus et accusés de ceux en appel ou en pourvoi. Cependant, ces données sont trop grossières pour permettre une analyse de l'utilisation, par les mineurs, des voies de recours. Il n'est même pas possible de distinguer les appels "parquet» des appels des condamnés. En revanche, comme précédemment, examiner le détail statistique des jeunes condamnés à moins de six mois par rapport à celui des adolescents en attente de transfert permet d'obtenir quelques renseignements supplémentaires. 
Graphique 9 : Entrées en maison d'arrêt, de justice et de correction des mineurs condamnés à moins de six mois d'emprisonnement et des mineurs en attente de leur transfert (1910-1945)

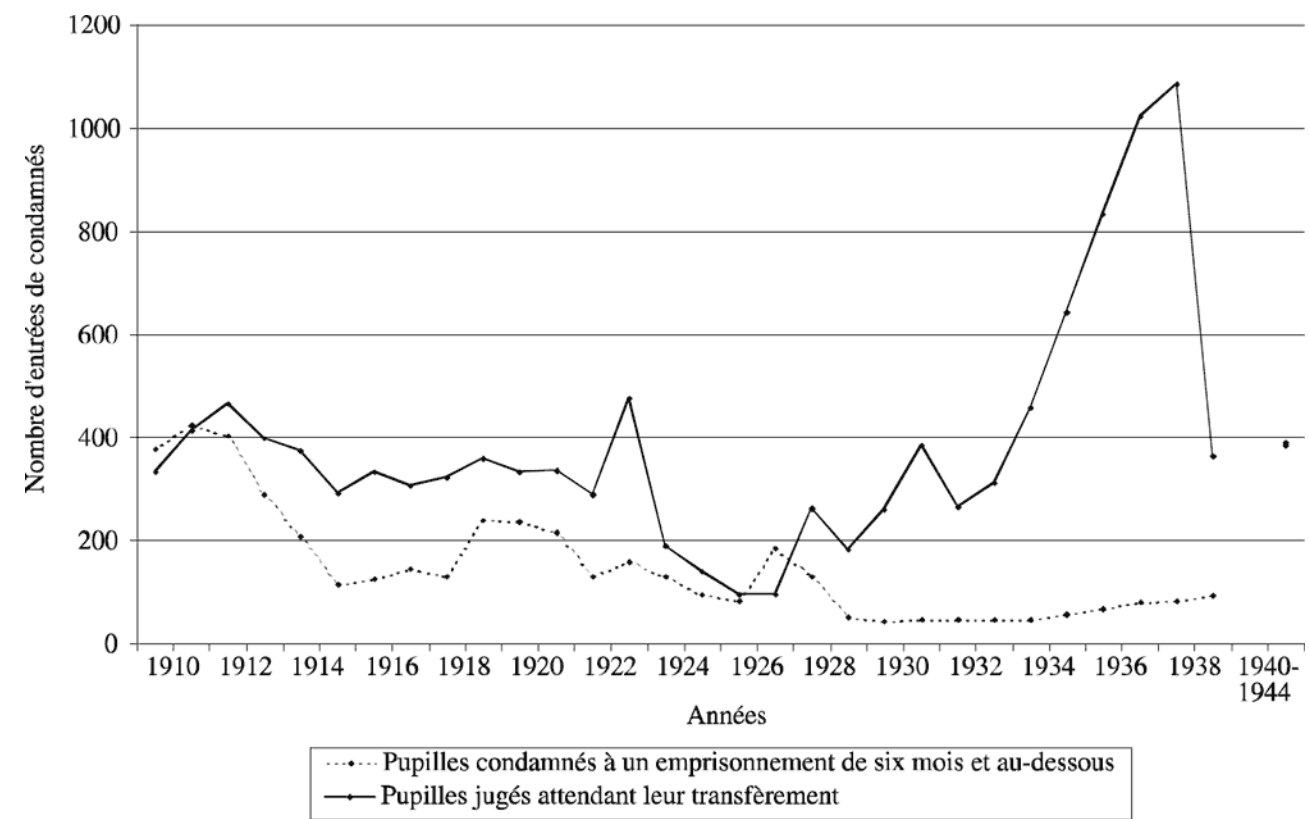

De 1910 à 1945, les mineurs en attente de transfèrement sont généralement plus nombreux à être détenus en maisons d'arrêt que ceux condamnés à une peine de six mois d'emprisonnement ou au dessous. Des exceptions sont à relever pour les années 1910 et 1927 où la tendance est inversée. La courbe consacrée aux jeunes en attente de transfèrement dans les lieux où ils doivent être détenus, soit en tant qu'acquittés, soit comme condamnés, est fort chaotique. Le pic qui se forme de 1933 à 1938 est particulièrement important puisque la différence est de 773 entrées supplémentaires entre ces deux années extrêmes et chute de 720 entrées pendant la seule année de 1939.

Les remarques faites pour la période précédente au sujet des statuts ambivalents des jeunes en attente de transfèrement sont toujours valables de 1910 à 1945. Les perturbations de la courbe des futurs transférés s'expliquent par les difficultés que le service affecté à cette tâche rencontre régulièrement :

[...] le service des transfèrements n'a pas fonctionné normalement par la suite de la continuation de l'état de guerre ${ }^{70}$.

Les départs des jeunes des maisons d'arrêt vers le patronage ou la colonie pénitentiaire auxquels ils ont été confiés sont donc parfois moins nombreux et laissent beaucoup d'adolescents longuement enfermés dans les cellules des prisons départementales. Par exemple, il est possible de supposer qu'en 1923, le service des voitures cellulaires a moins bien fonctionné qu'en 1924 puisque la courbe affiche un pic à cette période. De plus, de 1940 à 1944, malgré des données chiffrées inexistantes, il est prouvé que le service des transfèrements, comme lors de la Première Guerre mondiale, est défectueux. Plusieurs circulaires paraissent en effet à ce sujet, telle celle du 2 août 1940 :

En effet, en raison des circonstances actuelles, leur transport [celui des mineurs actuellement dans les maisons d'arrêt, en instance de conduite dans une institution publique d'Éducation surveillée], par mes services, peut être, suivant les cas, très difficile sinon impossible ${ }^{71}$.

47 Afin d'analyser la situation des mineurs "internés ", il faut avoir recours aux tableaux $\mathrm{n}^{\circ}$ I de la partie consacrée aux maisons d'arrêt, de justice et de correction, dans les 
volumes de Statistiques pénitentiaires, qui font état du «mouvement général des entrées et des sorties». En effet, les tableaux n ${ }^{\circ}$ II mêlent les enfants entrés par voie de correction paternelle et ceux venant de l'assistance publique. Il faut préciser que l'effectif redevient celui des détenus au 31 décembre de l'année. La lecture s'opère de nouveau en «stock». Il faut rappeler également que la courbe consacrée aux mineurs de la correction paternelle s'arrête inévitablement en 1935 puisque la loi est modifiée et qu'il n'est plus question alors d'incarcération dans les prisons départementales mais uniquement de détention dans les colonies pénitentiaires. La courbe des enfants venant de l'assistance publique se confond alors avec la courbe générale.

Graphique 10 : Les mineurs « internés » dans les maisons d'arrêt, de justice et de correction au 31 décembre de l'année (1910-1945)

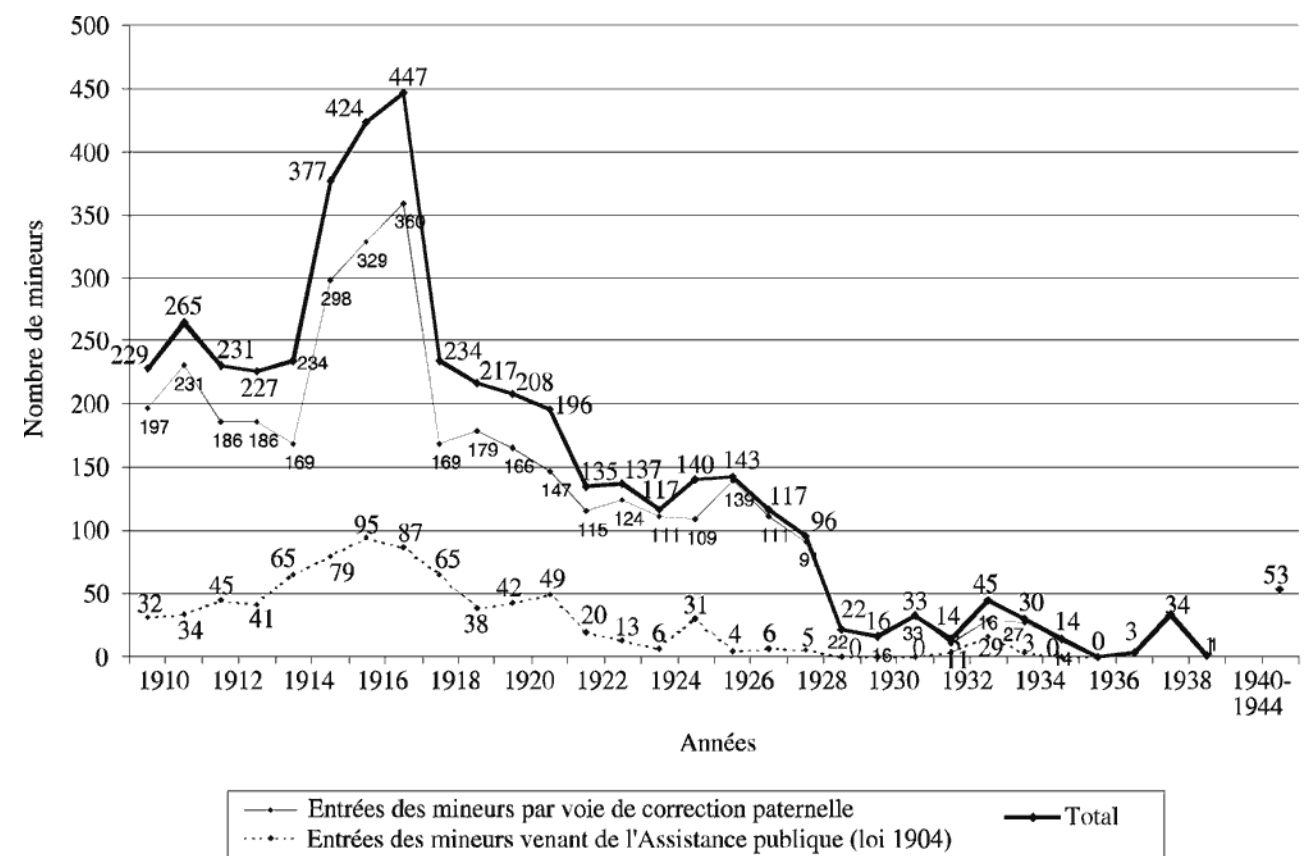

Ce graphique ne peut pas réellement révéler les pratiques liées aux mesures de détention administratives par voie de correction ou en vertu de la loi de 1904 pour les raisons déjà invoquées : le comptage en "stock » ne permet pas d'apprécier la quantité d'enfants qui passent, à l'année, par les prisons départementales puisqu'ils sont souvent détenus pour un court laps de temps. Cependant, une comparaison entre les deux types de détentions administratives est possible mais elle se borne à constater que les enfants venant de l'Assistance publique sont bien moins nombreux que ceux de la correction paternelle. L'usage de ces deux procédures civiles périclite vers la même période, dans l'immédiat après-guerre mais cela est un autre sujet.

\section{Conclusion}

Les statistiques et les courbes présentées mettent en évidence, à l'encontre de tous les discours réformateurs, le recours permanent de la détention en prison "ordinaire " pour les mineurs pénaux. D’abord moins utilisée que les «maisons de correction », elle devient dans l'entre-deux-guerres, une antichambre de ces dernières mais aussi un mode de punition en soit. De plus, l'enfermement en prison « ordinaire» ne fait pas 
nécessairement suite à une condamnation par les tribunaux correctionnels ou par les tribunaux pour enfants et adolescents. Parmi les mineurs incarcérés, ceux condamnés à une peine de prison ferme sont même minoritaires. Toutefois, les juges n'hésitent pas à prononcer de courtes peines de prison même si le discours philanthropique en souligne l'inutilité voire la nocivité. Jusqu'en 1935, les maisons d'arrêt, continuent de recevoir les enfants de la correction paternelle, même si l'usage de cette prérogative du pater familias tend à tomber en désuétude, du moins pour les garçons. Par contre, la pratique de la détention préventive d'intimidation, la prison " coup d'arrêt ", devient un mode courant de traitement des déviances juvéniles d'autant que les institutions de substitution à la prison sont peu nombreuses et régulièrement décriées ${ }^{72}$. Des mineurs incarcérés de quelques jours à deux-trois mois sont acquittés comme ayant agi sans discernement et remis à leurs parents sans aucune autre mesure. Le séjour derrière les barreaux tient alors lieu de processus de « rééducation » à lui seul. De 1880 à 1945, des dizaines de milliers d'enfants, souvent auteurs de délits sans grande gravité, parfois même innocents, se sont rencontrés sur les bancs de "l'école du vice ». Quant aux mineurs que les tribunaux confient aux institutions correctives, ils ont bien souvent attendu cette décision dans une cellule de prison départementale comme le montre la proportion des enfants entrés en "maison de correction » et provenant directement des maisons d'arrêt ${ }^{73}$. Le temps d'attente du jugement, le manque de place en colonies pénitentiaires et les difficultés de transfèrements prolongent leur expérience carcérale souvent plus longtemps qu'il n'est prévu dans les textes réglementaires.

Sur la période étudiée, les fréquentes réformes et les nombreux changements législatifs qui mettent en avant l'importance de la prévention, la protection de l'enfant davantage "victime" que "coupable " et qui visent à réduire le nombre des jeunes détenus, provoquent une certaine érosion de la population des mineurs incarcérés. Cependant, en France, la prison reste un dispositif central, un socle du traitement des mineurs de justice. Aussi, dès que la société prend peur de sa jeunesse, la courbe de la détention des mineurs à tendance à prendre un nouvel essor.

\section{BIBLIOGRAPHIE}

\section{Sources imprimées}

Collection des volumes de Statistique pénitentiaire. Exposé général de la situation des services et des divers établissements présenté à $M$. le garde des Sceaux par le directeur de l'Administration pénitentiaire et des services de l'Éducation surveillée, Melun, imprimerie administrative, 1880 à 1945 [sauf 1936, 1940-1945].

La guerre et la délinquance juvénile. Conférence d'experts réunis à Genève du 29 avril au 2 mai 1947 au Secrétariat de l'Union internationale de protection de l'enfance, Revue internationale de l'enfant, 1947, 11, 2-3, pp. 51-183. 
Actes du colloque national : autour du centenaire de la loi du 24 juillet 1889 relative à la protection des enfants maltraités et moralement abandonnés,Archives d'Aquitaine de recherche sociale, numéro spécial, 1989-1990.

Circulaire du 2 août 1940, Code pénitentiaire, tome XXVIII, Instruction n $36^{\text {quintième }}$.

Circulaire du 8 mars 1884, relative au transfèrement des jeunes détenus condamnés à l'emprisonnement en application de l'article 67 du code pénal, Code pénitentiaire, tome IX.

Décret du 6 août 1914, autorisant l'engagement des jeunes gens de 17 ans pour la durée de la guerre, Journal Officiel du27-2-1914.

Haussonville G. Y. d' (dir.), Enquête parlementaire sur le régime des établissements pénitentiaires, 8 tomes, Paris, Imprimerie nationale, 1873-1875.

Loi du 12 avril 1906, modifiant les articles 66 et 67 du code pénal, 340 du Code d'instruction criminelle, et fixant la majorité pénale à l'âge de 18 ans, Code pénitentiaire, tome XVII.

Loi du 15 avril 1892, imputant la détention préventive sur la durée des peines prononcées, Journal Officiel du17-11-1892.

Loi du 19 avril 1898 sur la répression des violences, voies de fait, actes de cruauté et attentats commis envers les enfants, Code pénitentiaire, tome XV.

Loi du 19 avril 1898, sur la répression des violences, voies de fait, actes de cruauté et attentats commis envers les enfants, Code pénitentiaire, tome XV.

Loi du 24 juillet 1889 sur la protection des enfants maltraités ou moralement abandonnés (déchéance de la puissance paternelle), Journal Officiel du 25-7-1889.

\section{Bibliographie}

Anonyme, La guerre et la délinquance juvénile, Revue internationale de l'enfant, 1947, 11, 2-3, pp. 51-183.

Bourquin J., Pierre É., La colonie agricole de Mettray, Sociétés \& représentations, 1996, 3, pp. 205-217.

Chauvière M., Lenoël P., Pierre É., (dir.), Protéger l'enfant. Raison juridique et pratiques sociojudiciaires XIX ${ }^{e}-X^{e}$ siècles, Rennes, PUR, 1996.

Corbin A., Les filles de noces. Misère sexuelle et prostitution au XIX ${ }^{e}$ siècle, Paris, Champs Flammarion, 1982.

Cousin L., Les Apaches, délinquance juvénile à Paris au début du XXe siècle, maîtrise d'histoire, Paris VII, 1976.

Darmon P., Vivre à Paris pendant la Grande Guerre, Paris, Fayard, 2002.

Dessertine D., Maradan B., L'âge d'or des patronages (1919-1939). La socialisation de l'enfance par les loisirs, Paris, Vaucresson CNFE-PJJ, 2001.

Dupont-Bouchat M.-S., Pierre É. (dir.), Enfance et justice au XIX ${ }^{e}$ siècle. Essais d'histoire comparée de la protection de l'enfance, 1820-1914, Paris, PUF, 2001.

Farcy J.-C., L'histoire de la justice française de la Révolution à nos jours, Paris, PUF, 2001.

Gaillac H., Les maisons de correction, 1830-1945, Paris, Cujas, 1971, $2^{\mathrm{e}}$ éd., 1991. 
Hamelin F., Femmes dans la nuit. L'internement à la Petite Roquette et au camp des Tourelles (1939-1944), Luisant, Renaudot et $\mathrm{C}^{\mathrm{ie}}, 1988$.

Laorde A., Les mineurs et la loi pénale. Commentaire de la loi du 12 avril 1906 (Loi Cruppi), Lois Nouvelles. Première partie. Revue de législation, $25^{\text {e }}$ année, 1906, pp. 305-326.

Leroy I., Le système correctionnel pour jeunes filles de 1871 à 1914, mémoire de maitrise d'histoire, Paris VII, 1988.

Merle P., La démocratisation de l'enseignement, Paris, La découverte, 2002.

Michard H., La délinquance des jeunes en France, Paris, Documentation française, 1978.

Mucchielli L. (dir.), Histoire de la criminalité française, Paris, l'Harmattan, 1994.

Ory P., La belle illusion. Culture et politique sous le signe du Front populaire, 1935-1938, Paris, Plon, 1994.

Perrot M., Les enfants de la Petite Roquette, l'Histoire, 1987, 100, pp. 30-38.

Perrot M., Des Apaches aux Zoulous... ou de la modernité des Apaches, Droit de l'enfance et de la famille, ministère de la Justice, Cahiers, 1996, pp. 49-54.

Pierre É., Débats pénitentiaires, politiques correctionnelles et vote de la loi de 1850, in Chauvière M., Lenoël P., Pierre É. (dir.), Protéger l'enfant. Raison juridique et pratiques socio-judiciaires $\mathrm{XIX}^{e}-\mathrm{XX}^{e}$ siècles, Rennes, PUR, 1996, pp. 71-105.

Quétel C., La syphilis - Présentation, in Bardet J.-P. (dir.), Peurs et terreurs face à la contagion, Paris, Fayard, 1988, pp. 285-295.

Quincy-Lefebvre P., Famille, institutions et déviance. Une histoire de l'enfance difficile, 1880-fin des années trente, Paris, Economica, 1997.

Schnapper B., La correction paternelle et le mouvements des idées au XIX ${ }^{\mathrm{e}}$ siècle, Revue historique, avril-juin 1980, pp. 321-349.

Stora-Lamarre A., Du sursis à l'exécution des peines : les fondements doctrinaux de la loi du 26 mars 1891, in Garnot B. (dir.), Ordre moral de la délinquance de l'Antiquité au XX ${ }^{e}$ siècle, Actes du colloques de Dijon, 7-8 octobre 1993, Dijon, Édition universitaire de Dijon, 1994, pp. 225-232.

Sudan D., De l'enfant coupable au sujet de droits : changements dans les dispositifs de gestion de la déviance juvénile (1820-1989), Déviance et société, 1997, 21, 4, pp. 383-400.

Thébaud F., La grande guerre, in Duby G., Perrot P. (dir.), Histoire des femmes en occident. Le XX siècle, tome 5, Paris, Tempus, 1992, pp. 85-144.

Vimont J.-C., La prison à l'ombre des hauts murs, Paris, Gallimard, 2004.

Yvorel É., À la marge des prisons pour mineurs : les prisons-écoles, des structures carcérales à vocation éducative et professionnalisante ", Revue d'histoire de l'enfance "irrégulière", décembre 2005, 7, pp. 17-40.

Yvorel É., Les enfants de l'ombre : la vie quotidienne des jeunes détenus au XXe siècle, Rennes, PUR, 2007.

Yvorel É., Les “blousons noirs” mineurs et l'Éducation surveillée : la répression d'un mythe, in Mohammed M., Mucchielli L. (dir.), Les bandes de jeunes, des blousons noirs à nos jours, Paris, La découverte, 2007, pp. 39-60.

Yvorel J.-J. (dir.), Cent ans de répression des violences à enfants, Le temps de l'histoire, 1999.

Yvorel J.-J., De l'enfance coupable à l'enfance victime. Les limites de la générosité philanthropique, in Garnot B. (dir.), Les victimes, des oubliés de l'histoire?, Actes du colloque de Dijon, Rennes, PUR, 2000, pp. 87-94. 


\section{NOTES}

2. Farcy (2001).

3. Bourquin, Pierre (1996).

4. Ory (1994).

5. Les colonies pénitentiaires sont en effet généralement spécialisées dans l'un de ces domaines. Les plus emblématiques des colonies publiques sont Saint-Maurice pour la formation industrielle, Saint-Hilaire pour l'apprentissage agricole et Belle-Île-en-Mer est l'unique colonie maritime.

6. Cette prison reçoit les jeunes garçons de justice jugés par les tribunaux parisiens de 1832 à 1930. Perrot (1987).

7. Yvorel É. (2007).

8. Statistique pénitentiaire pour l'année 1910 (1912, p. 102).

9. Cf. reproductions d'extraits des tableaux n II de 1895 et de 1911.

10. Malgré son titre, la loi de 1906 ne modifie guère l'article $67 \mathrm{du}$ code pénal. Cette dénomination est destinée à attirer l'attention sur le fait que les jeunes concernés par l'article 67 qui tend à atténuer les peines encourues par les mineurs, sont uniquement ceux de moins de 16 ans et non pas, malgré le changement de majorité pénale, ceux de 18 ans.

11. Loi du 12 avril 1906, modifiant les articles 66 et 67 du code pénal, Code pénitentiaire, tome XVII.

12. Rupture marquée sur les graphiques par l'axe vertical en pointillés.

13. Mucchielli (dir.) (1994).

14. Haussonville (1873-1875, p. 287).

15. Merle (2002).

16. Dessertine, Maradan (2001).

17. Vimont (2004, p. 48).

18. Gaillac ( $2^{\text {ème }}$ ed. 1991).

19. Pour les garçons, les Statistiques pénitentiaires dénombrent treize établissements publics en 1894 et il ne subsiste que huit établissements privés à la même date.

20. Au 31 décembre 1894, les treize établissements publics pour garçons comptent un total de 2541 pupilles.

21. Dupont-Bouchat, Pierre (dir.) (2001).

22. Chauvière, Lenoël, Pierre (dir.) (1996).

23. Loi du 24 juillet 1889 sur la protection des enfants maltraités, Journal Officiel du25-7-1889.

24. Loi du 19 avril 1898 sur la répression des violences, Code pénitentiaire, tome XV.

25. Stora-Lamarre (1994).

26. Pour l'année 1906, les Statistiques pénitentiaires comptent 15 garçons entrés en établissements d'éducation correctionnelle publics et privés en vertu de la loi de 1904 sur un total de 1682 entrées.

27. Circulaire du 8 mars 1884, relative au transfèrement des jeunes détenus, Code pénitentiaire, tome IX.

28. En 1900, sur 1731 entrées en établissements publics et privés au cours de l'année, 618 garçons viennent directement « des prisons du département où ils ont été jugés ».

29. Aire hachurée.

30. Quétel (1988).

31. Puis à Fresnes à partir de 1902.

32. Corbin (1982).

33. Les jeunes filles affectées de maladies vénériennes ou enceintes demeurent à Saint-Lazare. Quincy-Lefebvre, (1997, p. 153); Leroy (1988).

34. Au 31 décembre 1899, elles sont 18 pupilles à Nanterre contre 90 en 1894.

35. Statistique pénitentiaire pour l'année 1906 (1908, p. 100). 
36. En revanche, pour une étude régionale, qui porte donc sur des chiffres plus petits, il aurait fallu avoir recours aux registres d'écrou.

37. Yvorel É. (2007).

38. Cette courbe est établie à partir des mêmes tableaux noII des rapports annuels de Statistiques pénitentiaires décrits et utilisés précédemment pour l'analyse de la population des détenus mineurs.

39. Perrot (1996); Cousin (1976).

40. Chauvière, Lenoël, Pierre (dir.) (1994).

41. Décret du 6 août 1914, autorisant l'engagement des jeunes gens, Journal officiel du 27-2-1914.

42. Darmon (2002, p. 104).

43. Statistiques pénitentiaires pour l'année 1915 (1917, p. 6).

44. Au 31 décembre 1910, 3563 garçons sont enfermés en colonies publiques et privées contre 2315 en décembre 1914.

45. Vimont (2004, p. 52).

46. Darmon (2002, p. 103).

47. Vimont (2004, p. 52).

48. Chauvière, Lenoël, Pierre (dir.) (1994).

49. Toutefois, l'article 66 du code pénal est largement utilisé afin d'envoyer les petits vagabonds en correction.

50. Archives de Vaucresson, FH/BR. 31.908, «Rapport présenté par l'inspection générale des services administratifs », Melun, Impr. administrative, 1932, p. 120.

51. Yvorel É. (2007).

52. Archives de Vaucresson, $F H / B R$. 31.908, «Rapport présenté par l'inspection générale des services administratifs », Melun, Impr. administrative, 1932, p. 120.

53. Deux grandes campagnes de presse vindicatives ont en effet dénoncé les conditions d'enfermement exécrables des colons des "maisons de correction", la première en 1924 à l'instigation du journaliste Louis Roubaud, la seconde dix ans plus tard sur l'initiative d'Alexis Danan qui dénonce les « bagnes pour enfants».

54. Par exemple, le quartier pour mineurs de Fresnes (nommé Maison d'Éducation surveillée bien qu'installé au cœur des prisons de Fresnes) accueille des médecins à partir de 1936.

55. Il en est de même dans les institutions publiques d'Éducation surveillée avec 1392 entrées au cours de l'année 1945.

56. Michard (1978).

57. Anonyme, 1947.

58. Thébaud (1992).

59. Hamelin (1988). Là encore, ces différentes hypothèses sont confirmées par les données relatives aux Écoles de préservation : 297 entrées en 1920 (dont 222 venant de maisons d'arrêt), 268 entrées en 1925 (dont 153 venant de maisons d'arrêt), 301 entrées en 1931 (dont 168 venant de maisons d'arrêt), 172 entrées en 1935 (dont 76 venant de maisons d'arrêt), 30 entrées en 1939 (dont 20 venant de maisons d'arrêt).

60. Loi du 12 août 1850, sur l'éducation et le patronage des jeunes détenus, Bulletin des lois, CCCI, $n^{\circ} 2$ 342, art. 4. Pierre (1996, pp. 71-105).

61. Schnapper (1980, p. 536).

62. Ibid., p. 53.

63. Loi du 15 avril 1892, imputant la détention préventive sur la durée des peines prononcées, Journal Officiel du17-11-1892.

64. Archives du CARAN, BB ${ }^{18} 6004^{2}$ ( 4 BL 2 à 23 ) : Détention préventive, "Correspondance entre le président du Conseil et le ministre de la Justice au sujet de l'application de la loi du 15 novembre 1892 », 19 octobre 1907 et 29 octobre 1907. Les noms ont été changés.

65. Loi du 19 avril 1898, sur la répression des violences, Code pénitentiaire, tome XV. 
66. Yvorel J.-J. (2000).

67. Archives départementales de la Vienne, 2U, Tribunaux, prison de Châtellerault; 2U2518, Jugement correctionnel, « Registre de 1898, audience du 22 mars 1898 ». Les noms ont été changés.

68. La mise en forme du registre est respectée. Figure en gras, le texte préimprimé; en gris et italique, les inscriptions manuscrites; et en gris non italique le résumé des informations. À noter que la mention «détenu», lorsqu'elle n'est pas barré signifie que l'enfant est en détention préventive dans la maison d'arrêt du tribunal.

69. Chiffre atteint en 1912. Rappelons qu'il n'est pas possible de prendre en compte le chiffre de 1936 puisque, faute de la disponibilité du rapport, il est une moyenne entre les données des années 1935 et 1937.

70. Statistique pénitentiaire pour l'année 1915 (1917, p. 12).

71. Circulaire du 2 août 1940, Code pénitentiaire, tome XXVIII.

72. Sudan (1997, p. 389).

73. Selon les époques, entre un tiers et la moitié des garçons des colonies sont des transférés des prisons départementales.

\section{RÉSUMÉS}

L'historiographie de la répression de la délinquance juvénile laisse penser que seules les «maisons de correction» ont accueilli les mineurs de justice. Cet article rappelle, par l'analyse systématique des statistiques officielles de l'Administration pénitentiaire, que la prison « ordinaire » reçoit constamment nombre d'enfants condamnés, prévenus et de la correction paternelle.

$\mathrm{Au}$ delà des constats quantitatifs, l'auteure propose une interprétation des courbes de la détention des filles et garçons par le biais du double contexte politique et social et des législations qui régissent, progressivement, la protection de l'enfant délinquant considéré comme « victime » et non plus comme « coupable».

The historiography of juvenile delinquency repression gives the impression that reformatories were the only places to «host» minors. This article, through the systematic analysis of the penitentiary administration official statistics, shows that «ordinary» prisons also house a large number of convicted minors.

The author goes beyond the mere numbers and gives an interpretation of the detention of girls and boys graphics through the political and social context. She also analyses laws which, more and more often, deal with juvenile delinquents as «victims» and not simply «guilty».

\section{AUTEUR}

\section{ÉLISE YVOREL}

217, rue de Tolbiac, 75013 Paris, yvorel.e@free.fr

L'auteure est Docteure en histoire contemporaine de l'université de Poitiers et Chercheuse associée au laboratoire GERHICO de Poitiers. Ses principales publications : Les enfants de l'ombre : la vie quotidienne des jeunes détenus au XX siècle, Rennes, PUR, 2007; À la marge des prisons pour 
mineurs : les prisons-écoles, des structures carcérales à vocation éducative et professionnalisante ", Revue d'histoire de l'enfance "irrégulière”, décembre 2005, 7, pp. 17-40; « Les "blousons noirs" mineurs et l'Éducation surveillée : la répression d'un mythe ", in Mohammed M., Mucchielli L. (dir.), Les bandes de jeunes, des blousons noirs à nos jours, Paris, La découverte, 2007, pp. 39-60. Sa recherche en cours porte sur L'enfermement des mineurs délinquants : une comparaison européenne (1945-2005), qui est une comparaison du traitement judiciaire des jeunes délinquants en Allemagne, en Angleterre, en Belgique et en France. 